\title{
Homogenization of linear hyperbolic stochastic partial differential equation with rapidly oscillating coefficients: The two scale convergence method
}

\author{
Mogtaba Mohammed and Mamadou Sango * \\ Department of Mathematics and Applied Mathematics, University of Pretoria, Pretoria 0002, South \\ Africa \\ E-mails:mogtaba@aims.ac.za, sango7777@yahoo.com
}

*Corresponding author. E-mail: sango7777@yahoo.com.

\begin{abstract}
In this paper we establish new homogenization results for stochastic linear hyperbolic equations with periodically oscillating coefficients. We first use the multiple expansion method to drive the homogenized problem. Next we use the two scale convergence method and Prokhorov's and Skorokhod's probabilistic compactness results. We prove that the sequence of solutions of the original problem converges in suitable topologies to the solution of a homogenized stochastic hyperbolic problem with constant coefficients. We also prove a corrector result.
\end{abstract}

Keywords: homogenization, two-scale convergence, hyperbolic stochastic PDE, corrector result, Prokhorov and Skorokhod compactness results

\section{Introduction}

Homogenization is a mathematical theory aimed at understanding the behavior of processes that take place in heterogeneous media with highly oscillating heterogeneities at the microscopic level using properties of the homogeneous media obtained by homogenizing these materials. These heterogeneous materials consist of finely mixed different components like soil, paper, concrete for building, fibreglass, materials used in the manufacturing of high tech equipments such as planes, rockets and so on. This signifies that almost everything around us in real life is a heterogeneous material. The physical problems described on heterogeneous materials such as heat, mechanical constraints, flow of fluids in these media leads to the study of PDEs with highly oscillating coefficients depending on macroscopic scales or boundary value problems for PDEs in domain with fine grained boundaries. The main obstacle in solving these problems arises either from the character of the domain or the presence of high oscillations in the coefficients of the governing equation. To this end, it is expensive to compute solutions to these type of problems. Numerical methods have proved inefficient in solving such problems due to the fact that even the most advanced parallel computers are unable to simulate schemes related to the physically interesting such problems. 
The study of homogenization for PDEs in periodic structures has been undertaken by many authors. It was originally based on the idea of asymptotic expansions in powers of the small perturbation parameter in the problem. This approach was fundamental in the celebrated work [9] of Bensoussan, Lions and Papanicolaou; we should also mention the monograph by Bakhvalov and Panasenko [6]. These authors studied wide range of partial differential equations, such as elliptic, parabolic and hyperbolic problems, mainly linear in structure. The energy method of Tartar [27,51] introduced in 1977 by his construction a suitable oscillating test functions to study the homogenization of boundary value problems in the periodic setting. A great wealth of interesting results were obtained by many mathematicians, it will not be possible to survey most of these results, some of which may be found for instance in $[3,5,16,17,21$, $28,31,41,42,53]$.

In 1989, Nguetseng [28] introduced a general convergence result to study the homogenization of boundary value problem with periodic rapidly oscillating coefficients. What makes the convergence of Nguetseng so revolutionary in the field of homogenization is that, the weak limit he obtained depends on two variables, the additional variable is a reflection of the micro oscillations in the sequence, which is not captured in the classical weak limits. In 1992, Allaire [3,4] named the convergence of Nguetseng by the two scale convergence and further developed and investigated the properties of the two scale convergence. He introduced several types of admissible oscillating test functions and he also applied the two scale convergence to the homogenization of linear and nonlinear boundary value problems. It should be noted that the two scale convergence provides a rigorous mathematical justification of the heuristic method of asymptotic expansions. In 1994, the two scale convergence was further extended from the periodic to the random setting by Bourgeat, Mikelić and Wright [12] under the name of "Stochastic two-scale convergence". Recently two scale convergence has been generalized to homogenization problems on nonperiodic algebras, see for instance [29,30,46] and [48]. We also note the newly introduced unfolding method by Cioranescu, Damlamian and Griso in $[14,15]$.

In view of the prevalence of randomness in almost all natural phenomena, it was not long before homogenization of PDEs with random coefficients started to be investigated. Pioneers in this direction are certainly Kozlov [24], Papanicolaou and Varadhan [34]. Their work influenced many new research; see for instance $[12,22,26,36,47,50]$.

As mentioned above, there was a need to consider homogenization of PDEs with random coefficients. However physical processes under random fluctuations are better modelled by stochastic partial differential equations (SPDEs). It was therefore natural to consider homogenization of this very important class of PDEs. Research in this direction is still at its infancy, despite the importance of such problems in both applied and fundamental sciences. Some relevant interesting work have recently been undertaken, mainly for parabolic SPDEs, see for instance [7,19,37,40,43,45].

The homogenization of hyperbolic SPDEs has not been considered so far. The main aim of the present work is to initiate such investigation. As far as the homogenization of deterministic hyperbolic (PDEs) is concerned, many work have been undertaken by several authors from different perspectives. We refer to [9] where the authors studied the homogenization of the hyperbolic equations based on asymptotic expansions. We also note the monograph of Cioranescu and Donato [17], where similar studies are carried through in the framework of Tartar's method, which was introduced in [27,51]. Cioranescu and Donato also proved the convergence of the energy associated to the inhomogeneous wave equation to the energy associated to the homogenized problem; the corresponding corrector result was proved in [13]. Recently, the new field of numerical homogenization is attracting a growing attention of researchers in applied mathematics. Some numerical works have considered wave equations in heterogeneous media using finite element heterogeneous multiscale method [1,2] and the upscaling method $[11,23,33]$. It 
would be of interest to investigate homogenization of hyperbolic SPDEs in the framework of these methods in our future work.

In this work we will be concerned with establishing homogenization results for linear hyperbolic equations with periodically oscillating coefficients in the framework of the multiple expansion method which is formal and widely used in physics and mechanics. Our main result is to adapt the two scale convergence method to our problem. Two scale convergence is an outstanding approach in proving the homogenization result as well as in obtaining the corrector result.

We study the asymptotic behaviour of solutions $u^{\epsilon}=u^{\epsilon}(\omega, x, t)$ of the initial boundary value problem with oscillating data:

$$
\begin{cases}\mathrm{d} u_{t}^{\epsilon}=\operatorname{div} A_{\epsilon} \nabla u^{\epsilon} \mathrm{d} t+f^{\epsilon} \mathrm{d} t+g^{\epsilon} \mathrm{d} W & \text { in } Q \times(0, T), \\ u^{\epsilon}=0 & \text { on } \partial Q \times(0, T), \\ u^{\epsilon}(x, 0)=a^{\epsilon}(x), \quad u_{t}^{\epsilon}(x, 0)=b^{\epsilon}(x), & \end{cases}
$$

where $\epsilon>0$ sufficiently small, $T>0, Q$ is an open bounded (at least Lipschitz) subset of $\mathbb{R}^{n}, W=$ $(W(t))_{0 \leqslant t \leqslant T}$ an $m$-dimensional standard Wiener process defined on a given filtered complete probability space $\left(\Omega, \mathcal{F}, \mathbb{P},\left(\mathcal{F}_{t}\right)_{0 \leqslant t \leqslant T}\right) ; \mathbb{E}$ denote the corresponding mathematical expectation, $f^{\epsilon}(x, t)=f\left(\frac{x}{\epsilon}, t\right)$, $g^{\epsilon}(x, t)=g\left(\frac{x}{\epsilon}, t\right), a^{\epsilon}(x)=a\left(\frac{x}{\epsilon}\right), b^{\epsilon}(x)=b\left(\frac{x}{\epsilon}\right)$ and $A_{\epsilon}(x)=A\left(\frac{x}{\epsilon}\right)=\left(a_{i, j}\left(\frac{x}{\epsilon}\right)\right)_{1 \leqslant i, j \leqslant n}$ an $n \times n$ symmetric matrix such that

(A1) $\sum_{i, j=1}^{n} a_{i, j} \xi_{i} \xi_{j} \geqslant \alpha \sum_{i=1}^{n} \xi_{i}^{2}$ for all $\xi \in \mathbb{R}^{n}$ and $\alpha$ is a positive constant,

(A2) $a_{i, j} \in L^{\infty}\left(\mathbb{R}^{n}\right), i, j=1, \ldots, n$,

(A3) $a_{i, j}$ are $Y$-periodic $\forall i, j=1, \ldots, n$.

The differential $g^{\epsilon} \mathrm{d} W$ is understood in the sense of Itô.

Problems of type $\left(P_{\epsilon}\right)$ arise in several physical phenomena in the presence of random fluctuations, for instance, in the modeling of waves generated in a vibrating string, an elastic membrane and a rubbery solid in dimensions 1,2 and 3, respectively. To illustrate that, for example let us consider the disturbance generated in bridge cables. These cables are made up of composite materials and vibrate continuously with high irregularity as a response to wind blow. In this case the external force is given by $g^{\epsilon}(t, x) \mathrm{d} W$. See Fig. 1. It is also possible that the disturbance arises via other sources such as birds landing on or taking off from the cable. In this case, the intensity of the disturbance on the cable is moderate. Thus, the force has a more regular behaviour and therefore, the stochastic term may be neglected. In this case, the force is represented by $f^{\epsilon}(t, x)$. In fact problem $\left(P_{\epsilon}\right)$ can also be understood according to the well-known Walsh interpretation [54]. It is clear that the strings of a guitar have the structure of a composite material.

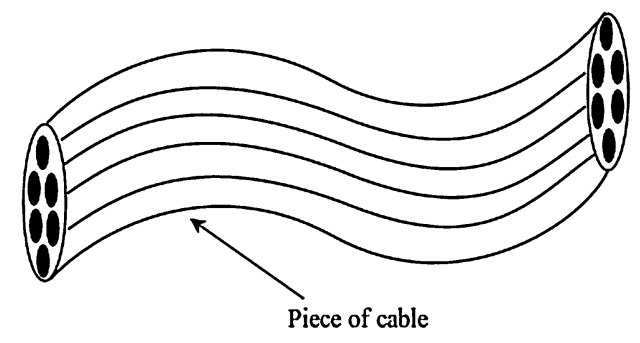

Fig. 1. 
When bombarded by particles of sand, the motion of the strings is subjected to random vibrations. Such a process can be modeled by problem $\left(P_{\epsilon}\right)$. Wave equations in heterogeneous media have applications in several other branches of science such as geoscience, physics and engineering [11,20].

In order to state some facts we need to introduce some spaces. We consider the well-known spaces $L^{2}(Q), H^{1}(Q), H_{0}^{1}(Q), C_{\text {per }}^{\infty}(Y)$ is the subspace of $C^{\infty}\left(\mathbb{R}^{n}\right)$ of $Y$-periodic functions where $Y=\left(0, l_{1}\right) \times$ $\cdots \times\left(0, l_{n}\right)$. Let $H_{\text {per }}^{1}(Y)$ be the closure of $C_{\text {per }}^{\infty}(Y)$ in the $H^{1}$-norm, and $H_{\text {per }}(Y)$ the subspace of $H_{\text {per }}^{1}(Y)$ with zero mean on $Y$.

For a Banach space $X$, and $1 \leqslant p, q \leqslant \infty$, we denote by $L^{p}(0, T ; X)$ the space of measurable functions $\phi: t \in[0, T] \rightarrow \phi(t) \in X$ such that $\|\phi(t)\|_{X} \in L^{p}(0, T)$ and by $L^{q}\left(\Omega, \mathcal{F}, \mathbb{P} ; L^{p}(0, T ; X)\right)$ we denote the space of functions $\phi:(\omega, t) \in \Omega \times[0, T] \rightarrow \phi(\omega, t, \cdot) \in X$ such that $\phi(\omega, t, x)$ is measurable with respect to $(\omega, t)$ and for each $t$ is $\mathcal{F}_{t}$-measurable in $\omega$, we endow the later space with the norm

$$
\|\phi\|_{L^{q}\left(\Omega, \mathcal{F}, \mathbb{P} ; L^{p}(0, T ; X)\right)}=\left(E\|\phi\|_{L^{p}(0, T ; X)}^{q}\right)^{1 / q} .
$$

When $p=\infty$, the space

$$
L^{\infty}(0, T ; X)=\left\{\phi:[0, T] \rightarrow X \text { such that ess } \sup _{X}\|\phi\|_{X}<\infty\right\},
$$

where ess $\sup _{X}\|\phi\|_{X}=\|\phi\|_{L^{\infty}(0, T ; X)}$. When $p=\infty$, we endow $L^{q}\left(\Omega, \mathcal{F}, \mathbb{P}, L^{\infty}(0, T ; X)\right)$ with the following norm

$$
\|\phi\|_{L^{q\left(\Omega, \mathcal{F}, \mathbb{P}, L^{\infty}(0, T ; X)\right)}}=\left(\mathbb{E}\|\phi\|_{L^{\infty}(0, T ; X)}^{q}\right)^{1 / q} .
$$

It is well known that, under the above norm, $L^{q}\left(\Omega, \mathcal{F}, \mathbb{P}, L^{p}(0, T ; X)\right)$ is a Banach space.

We shall often omit $\omega$ in the notation of $u_{\epsilon}$. In the following we introduce the notion of strong probabilistic solution for our problem.

Definition 1. We define the strong probabilistic solution of the problem $\left(P_{\epsilon}\right)$ as a process

$$
u^{\epsilon}: \Omega \times[0, T] \rightarrow H_{0}^{1}(Q),
$$

such that

(1) $u^{\epsilon}, u_{t}^{\epsilon}$ are continuous with respect to time in $H_{0}^{1}(Q), L^{2}(Q)$, respectively,

(2) $u^{\epsilon}, u_{t}^{\epsilon}$ are $\mathcal{F}_{t}$-measurable,

(3) $u^{\epsilon} \in L^{2}\left(\Omega, \mathcal{F}, \mathbb{P} ; L^{\infty}\left(0, T ; H_{0}^{1}(Q)\right)\right), u_{t}^{\epsilon} \in L^{2}\left(\Omega, \mathcal{F}, \mathbb{P} ; L^{\infty}\left(0, T ; L^{2}(Q)\right)\right)$,

(4) $\forall t \in[0, T], u^{\epsilon}(t, \cdot)$ satisfy

$$
\begin{aligned}
& \int_{0}^{t}\left(\mathrm{~d} u_{t}^{\epsilon}(t, \cdot), \phi\right) \mathrm{d} s+\int_{0}^{t}\left(A_{\epsilon} \nabla u^{\epsilon}(s, \cdot), \nabla \phi\right) \mathrm{d} s \\
& \quad=\int_{0}^{t}\left(f^{\epsilon}(s, \cdot) \phi\right) \mathrm{d} s+\left(\int_{0}^{t} g^{\epsilon}(s, \cdot) \mathrm{d} W(s), \phi\right) \quad \forall \phi \in C_{0}^{\infty}(Q) .
\end{aligned}
$$

The problem of existence and uniqueness of a strong probabilistic solution of $\left(P_{\epsilon}\right)$ was dealt with in [35]. The corresponding result follows. 
Theorem 1. Suppose that the assumptions (A1)-(A3) hold. Let

(A4) $a^{\epsilon} \in H_{0}^{1}(Q), b^{\epsilon} \in L^{2}(Q)$,

(A5) $f^{\epsilon} \in L^{2}(Q \times(0, T)), g^{\epsilon} \in L^{2}(Q \times(0, T))$.

Then for fixed $\epsilon>0$, the problem $\left(P_{\epsilon}\right)$ has a unique strong probabilistic solution

$$
u^{\epsilon} \in L^{2}\left(\Omega, \mathcal{F}, \mathbb{P} ; C\left([0, T] ; H_{0}^{1}(Q)\right)\right), \quad u_{t}^{\epsilon} \in L^{2}\left(\Omega, \mathcal{F}, \mathbb{P} ; C\left([0, T] ; L^{2}(Q)\right)\right),
$$

in the sense of Definition 1.

Our goals are described as follows: First, we show that the sequence of solutions $u^{\epsilon}$ converges in suitable sense as $\epsilon \rightarrow 0$ to a solution $u$ of the following stochastic partial differential equation (SPDE)

$$
\begin{cases}\mathrm{d} u_{t}=\operatorname{div} A_{0} \nabla u \mathrm{~d} t+f \mathrm{~d} t+g \mathrm{~d} W & \text { in } Q \times(0, T), \\ u=0 & \text { on } \partial Q \times(0, T), \\ u(x, 0)=a(x) \in H_{0}^{1}(Q), & u_{t}(x, 0)=b(x) \in L^{2}(Q),\end{cases}
$$

where $A_{0}$ is a constant elliptic matrix defined by

$$
A_{0}=\int_{Y}(A(y)-A(y) \chi(y)) \mathrm{d} y
$$

where $\chi(y) \in H_{\text {per }}(Y)$ is the unique solution of the following boundary value problem

$$
\left\{\begin{array}{l}
\operatorname{div}_{y}\left(A(y) \nabla_{y} \chi(y)\right)=\nabla_{y} \cdot A(y) \text { in } Y \\
\chi \text { is } Y \text { periodic }
\end{array}\right.
$$

for $Y=\left(0, l_{1}\right) \times \cdots \times\left(0, l_{n}\right)$. Next, we prove some corrector result.

This paper is organized as follows. In Section 2, we derive important a priori estimates that will be used in subsequent sections. Section 3 is devoted to the proof of the tightness of probability measures generated by the sequence of triples $\left(W, u^{\epsilon}, u_{t}^{\epsilon}\right)$; this will enable us to use Prokhorov's and Skorokhod's processes for the construction of a sequence of random variables $\left(W_{\epsilon_{j}}, u^{\epsilon_{j}}, u_{t}^{\epsilon_{j}}\right)$ defined on new probability spaces; $\left(W_{\epsilon_{j}}, u^{\epsilon_{j}}, u_{t}^{\epsilon_{j}}\right)$ satisfies the original problem $\left(P_{\epsilon}\right)$ and strongly converges in a suitable spaces to a triple $\left(\tilde{W}, u, u_{t}\right)$ that solve the homogenized problem $(P)$. In Section 4 we derive the homogenized problem using standard multiple expansion method. In the last section we introduce the two scale convergence and some of its properties, then in the first subsection we obtain the homogenization result using the two scale convergence method. We end the paper by proving a corrector result.

\section{The a priori estimates}

Here and in the sequel, $C$ will denote a constant independent of $\epsilon$. In this section we establish the a priori estimates announced earlier. In our first lemma, we prove that, both the solution to the problem $\left(P_{\epsilon}\right)$ and its time derivative are bounded in appropriate probabilistic evolution spaces. Likewise in our second lemma we establish a finite difference estimate of the time derivative of the solution in a space involving $H^{-1}(Q)$. 
Lemma 1. Under the assumptions (A1)-(A5), the solution $u^{\epsilon}$ of $\left(P_{\epsilon}\right)$ satisfies the following estimate

$$
\mathbb{E} \sup _{0 \leqslant t \leqslant T}\left\|u^{\epsilon}(t)\right\|_{H_{0}^{1}(Q)}^{2}+\mathbb{E} \sup _{0 \leqslant t \leqslant T}\left\|u_{t}^{\epsilon}(t)\right\|_{L^{2}(Q)}^{2} \leqslant C .
$$

Proof. The following arguments are used modulo appropriate stopping times. Itô formula and the symmetry of $A$ give

$$
d\left[\left\|u_{t}^{\epsilon}\right\|_{L^{2}(Q)}^{2}+\left(A_{\epsilon} \nabla u^{\epsilon}, \nabla u^{\epsilon}\right)\right]=2\left(f^{\epsilon}, u_{t}^{\epsilon}\right) \mathrm{d} t+2\left(g^{\epsilon}, u_{t}^{\epsilon}\right) \mathrm{d} W+\left\|g^{\epsilon}\right\|_{L^{2}(Q)}^{2} \mathrm{~d} t .
$$

Integrating over $(0, t), t \leqslant T$, we get

$$
\begin{aligned}
\left\|u_{t}^{\epsilon}(t)\right\|_{L^{2}(Q)}^{2}+\left(A_{\epsilon} \nabla u^{\epsilon}(t), \nabla u^{\epsilon}(t)\right)= & \left\|b^{\epsilon}\right\|_{L^{2}(Q)}^{2}+\left(A_{\epsilon} \nabla a^{\epsilon}, \nabla a^{\epsilon}\right) \\
& +2 \int_{0}^{t}\left(f^{\epsilon}, u_{t}^{\epsilon}\right) \mathrm{d} s+2 \int_{0}^{t}\left(g^{\epsilon}, u_{t}^{\epsilon}\right) \mathrm{d} W+\int_{0}^{t}\left\|g^{\epsilon}\right\|_{L^{2}(Q)}^{2} \mathrm{~d} s .
\end{aligned}
$$

Using the assumptions on the matrix $A$ and taking the supremum over $t \in[0, T]$, we have

$$
\begin{aligned}
& \sup _{0 \leqslant t \leqslant T}\left\|u_{t}^{\epsilon}(t)\right\|_{L^{2}(Q)}^{2}+\sup _{0 \leqslant t \leqslant T}\left\|u^{\epsilon}(t)\right\|_{H_{0}^{1}(Q)}^{2} \\
& \leqslant C\left\|b^{\epsilon}\right\|_{L^{2}(Q)}^{2}+C\left\|a^{\epsilon}\right\|_{H_{0}^{1}(Q)}^{2} \\
& \quad+2 C \int_{0}^{t}\left|\left(f^{\epsilon}, u_{t}^{\epsilon}\right)\right| \mathrm{d} s+2 C \int_{0}^{t}\left(g^{\epsilon}, u_{t}^{\epsilon}\right) \mathrm{d} W+C \int_{0}^{t}\left\|g^{\epsilon}\right\|_{L^{2}(Q)}^{2} \mathrm{~d} s .
\end{aligned}
$$

Taking the expectation on both sides, we have

$$
\begin{aligned}
& \mathbb{E}\left[\sup _{0 \leqslant t \leqslant T}\left\|u_{t}^{\epsilon}(t)\right\|_{L^{2}(Q)}^{2}+\sup _{0 \leqslant t \leqslant T}\left\|u^{\epsilon}(t)\right\|_{H_{0}^{1}(Q)}^{2}\right] \\
& \leqslant C \mathbb{E}\left[\left\|b^{\epsilon}\right\|_{L^{2}(Q)}^{2}+\left\|a^{\epsilon}\right\|_{H_{0}^{1}(Q)}^{2}+\int_{0}^{T}\left|\left(f^{\epsilon}, u_{t}^{\epsilon}\right)\right| \mathrm{d} t\right. \\
&\left.+\sup _{0 \leqslant t \leqslant T}\left|\int_{0}^{t}\left(g^{\epsilon}, u_{t}^{\epsilon}\right) \mathrm{d} W\right|+\int_{0}^{T}\left\|g^{\epsilon}\right\|_{L^{2}(Q)}^{2} \mathrm{~d} t\right] \\
& \leqslant C\left[C_{1}+\mathbb{E} \int_{0}^{T}\left|\left(f^{\epsilon}, u_{t}^{\epsilon}\right)\right| \mathrm{d} t+\mathbb{E} \sup _{0 \leqslant t \leqslant T}\left|\int_{0}^{t}\left(g^{\epsilon}, u_{t}^{\epsilon}\right) \mathrm{d} W\right|\right],
\end{aligned}
$$

where

$$
C_{1}=\left\|b^{\epsilon}\right\|_{L^{2}(Q)}^{2}+\left\|a^{\epsilon}\right\|_{H_{0}^{1}(Q)}^{2}+\int_{0}^{T}\left\|g^{\epsilon}\right\|_{L^{2}(Q)}^{2} \mathrm{~d} s
$$


Using Cauchy-Schwarz's and Young's inequalities, we have

$$
\begin{aligned}
\mathbb{E} \int_{0}^{T}\left(f^{\epsilon}, u_{t}^{\epsilon}\right) \mathrm{d} t & \leqslant \mathbb{E} \int_{0}^{T}\left\|f^{\epsilon}\right\|_{L^{2}(Q)}\left\|u_{t}^{\epsilon}\right\|_{L^{2}(Q)} \mathrm{d} t \leqslant \mathbb{E} \sup _{0 \leqslant t \leqslant T}\left\|u_{t}^{\epsilon}(t)\right\|_{L^{2}(Q)} \int_{0}^{T}\left\|f^{\epsilon}\right\|_{L^{2}(Q)} \mathrm{d} t \\
& \leqslant \varepsilon \mathbb{E} \sup _{0 \leqslant t \leqslant T}\left\|u_{t}^{\epsilon}(t)\right\|_{L^{2}(Q)}^{2}+C(\varepsilon)\left(\int_{0}^{T}\left\|f^{\epsilon}\right\|_{L^{2}(Q)}^{2} \mathrm{~d} t\right)^{2},
\end{aligned}
$$

where $\varepsilon>0$ is sufficiently small.

Thanks to Burkhölder-Davis-Gundy's inequality, followed by Cauchy-Schwarz's inequality, the second term in the right-hand side of (2) can be estimated as

$$
\mathbb{E} \sup _{0 \leqslant t \leqslant T}\left|\int_{0}^{t}\left(g^{\epsilon}, u_{t}^{\epsilon}\right) \mathrm{d} W\right| \leqslant C \mathbb{E}\left(\int_{0}^{T}\left(g^{\epsilon}, u_{t}^{\epsilon}\right)^{2} \mathrm{~d} t\right)^{\frac{1}{2}} \leqslant C \mathbb{E}\left(\int_{0}^{T}\left\|g^{\epsilon}\right\|_{L^{2}(Q)}^{2}\left\|u_{t}^{\epsilon}\right\|_{L^{2}(Q)}^{2} \mathrm{~d} t\right)^{\frac{1}{2}} .
$$

Again using Young's inequality, we get

$$
\begin{aligned}
C \mathbb{E}\left(\int_{0}^{T}\left\|g^{\epsilon}\right\|_{L^{2}(Q)}^{2}\left\|u_{t}^{\epsilon}\right\|_{L^{2}(Q)}^{2} \mathrm{~d} t\right)^{\frac{1}{2}} & \leqslant C \mathbb{E} \sup _{0 \leqslant t \leqslant T}\left\|u_{t}^{\epsilon}(t)\right\|_{L^{2}(Q)}^{2}\left(\int_{0}^{T}\left\|g^{\epsilon}\right\|_{L^{2}(Q)}^{2} \mathrm{~d} t\right)^{\frac{1}{2}} \\
& \leqslant C(\varepsilon) \mathbb{E} \sup _{0 \leqslant t \leqslant T}\left\|u_{t}^{\epsilon}(t)\right\|_{L^{2}(Q)}^{2}+\varepsilon C \int_{0}^{T}\left\|g^{\epsilon}\right\|_{L^{2}(Q)}^{2} \mathrm{~d} t
\end{aligned}
$$

where $\varepsilon>0$ is small enough. Using (3) and (4) into (2) and assumption (A5), we obtain

$$
\mathbb{E} \sup _{0 \leqslant t \leqslant T}\left\|u^{\epsilon}(t)\right\|_{H_{0}^{1}(Q)}^{2}+\mathbb{E} \sup _{0 \leqslant t \leqslant T}\left\|u_{t}^{\epsilon}(t)\right\|_{L^{2}(Q)}^{2} \leqslant C .
$$

The proof is complete.

Next we have the following lemma.

Lemma 2. Under the assumptions (A1)-(A5) with the replacement of the assumption on $g^{\epsilon}$ by $g^{\epsilon} \in$ $L^{4}\left((0, T) ; H^{-1}(Q)\right), u_{t}^{\epsilon}$ satisfies the following

$$
\mathbb{E} \sup _{|\theta| \leqslant \delta} \int_{0}^{T}\left\|u_{t}^{\epsilon}(t+\theta)-u_{t}^{\epsilon}(t)\right\|_{H^{-1}(Q)}^{2} \mathrm{~d} t<C \delta
$$

for any $\epsilon>0$ and sufficiently small $\delta>0$.

Proof. Assume that $u_{t}^{\epsilon}$ is extended by zero outside the interval $[0, T]$. We write

$$
u_{t}^{\epsilon}(t+\theta)-u_{t}^{\epsilon}(t)=\int_{t}^{t+\theta} \operatorname{div}\left(A_{\epsilon} \nabla u^{\epsilon}\right) \mathrm{d} s+\int_{t}^{t+\theta} f^{\epsilon} \mathrm{d} s+\int_{t}^{t+\theta} g^{\epsilon} \mathrm{d} W(s) .
$$


Then

$$
\begin{aligned}
\left\|u_{t}^{\epsilon}(t+\theta)-u_{t}^{\epsilon}(t)\right\|_{H^{-1}(Q)} \leqslant & \left\|\int_{t}^{t+\theta} \operatorname{div}\left(A_{\epsilon} \nabla u^{\epsilon}\right) \mathrm{d} s\right\|_{H^{-1}(Q)} \\
& +\left\|\int_{t}^{t+\theta} f^{\epsilon} \mathrm{d} s\right\|_{H^{-1}(Q)}+\left\|\int_{t}^{t+\theta} g^{\epsilon} \mathrm{d} W(s)\right\|_{H^{-1}(Q)} .
\end{aligned}
$$

Using assumption (A2), we have

$$
\begin{aligned}
\left\|\int_{t}^{t+\theta} \operatorname{div}\left(A_{\epsilon} \nabla u^{\epsilon}\right) \mathrm{d} s\right\|_{H^{-1}(Q)} & \leqslant \sup _{\phi \in H_{0}^{1}(Q):\|\phi\|=1}\left|\left\langle\int_{t}^{t+\theta} \operatorname{div}\left(A_{\epsilon} \nabla u^{\epsilon}\right) \mathrm{d} s, \phi\right\rangle_{H^{-1}(Q), H_{0}^{1}(Q)}\right| \\
& =\sup _{\phi \in H_{0}^{1}(Q):\|\phi\|=1} \int_{Q} \int_{t}^{t+\theta} A_{\epsilon} \nabla u^{\epsilon} \nabla \phi \mathrm{d} x \mathrm{~d} s \\
& \leqslant C \sup _{\phi \in H_{0}^{1}(Q):\|\phi\|=1} \int_{t}^{t+\theta}\left\|u^{\epsilon}\right\|_{H_{0}^{1}(Q)}\|\phi\|_{H_{0}^{1}(Q)} \mathrm{d} s \leqslant C \theta .
\end{aligned}
$$

From assumption (A5), we obtain

$$
\begin{aligned}
\left\|\int_{t}^{t+\theta} f^{\epsilon} \mathrm{d} s\right\|_{H^{-1}(Q)} & \leqslant \sup _{\phi \in H_{0}^{1}(Q):\|\phi\|=1}\left|\left\langle\int_{t}^{t+\theta} f^{\epsilon} \mathrm{d} s, \phi\right\rangle_{H^{-1}(Q), H_{0}^{1}(Q)}\right| \\
& =\sup _{\phi \in H_{0}^{1}(Q):\|\phi\|=1} \int_{Q} \int_{t}^{t+\theta} f^{\epsilon} \phi \mathrm{d} x \mathrm{~d} s \\
& \leqslant C \sup _{\phi \in H_{0}^{1}(Q):\|\phi\|=1} \int_{t}^{t+\theta}\left\|f^{\epsilon}\right\|_{L^{2}(Q)}\|\phi\|_{L^{2}(Q)} \mathrm{d} s \leqslant C \theta .
\end{aligned}
$$

Since

$$
\left\|\int_{t}^{t+\theta} g^{\epsilon} \mathrm{d} W(s)\right\|_{H^{-1}(Q)}^{2} \leqslant \sup _{\phi \in H_{0}^{1}(Q):\|\phi\|=1}\left|\left\langle\int_{t}^{t+\theta} g^{\epsilon} \mathrm{d} W(s), \phi\right\rangle_{H^{-1}(Q), H_{0}^{1}(Q)}\right|^{2},
$$

then Fubini's theorem gives

$$
\begin{aligned}
& \mathbb{E} \sup _{|\theta| \leqslant \delta} \int_{0}^{T}\left\|\int_{t}^{t+\theta} g^{\epsilon} \mathrm{d} W(s)\right\|_{H^{-1}(Q)}^{2} \mathrm{~d} t \\
& \leqslant \sup _{|\theta| \leqslant \delta} \int_{0}^{T} \sup _{\phi \in H_{0}^{1}(Q):\|\phi\|=1} \mathbb{E}\left(\int_{t}^{t+\theta}\left\langle g^{\epsilon}, \phi\right\rangle_{H^{-1}(Q), H_{0}^{1}(Q)} \mathrm{d} W(s)\right)^{2} \mathrm{~d} t .
\end{aligned}
$$

8 
Thanks to Burkhölder-Davis-Gundy's inequality, we get

$$
\begin{aligned}
& \sup _{|\theta| \leqslant \delta} \int_{0}^{T} \sup _{\phi \in H_{0}^{1}(Q):\|\phi\|=1} \mathbb{E}\left(\int_{t}^{t+\theta}\left\langle g^{\epsilon}, \phi\right\rangle_{H^{-1}(Q), H_{0}^{1}(Q)} \mathrm{d} W(s)\right)^{2} \mathrm{~d} t \\
& \quad \leqslant \sup _{|\theta| \leqslant \delta} \int_{0}^{T} \sup _{\phi \in H_{0}^{1}(Q):\|\phi\|=1} \int_{t}^{t+\theta}\left\langle g^{\epsilon}, \phi\right\rangle_{H^{-1}(Q), H_{0}^{1}(Q)}^{2} \mathrm{~d} s \mathrm{~d} t \leqslant \sup _{|\theta| \leqslant \delta} \int_{0}^{T} \int_{t}^{t+\theta}\left\|g^{\epsilon}\right\|_{H^{-1}(Q)}^{2} \mathrm{~d} s \mathrm{~d} t .
\end{aligned}
$$

But Cauchy-Schwarz's inequality gives

$$
\begin{aligned}
\sup _{|\theta| \leqslant \delta} \int_{0}^{T} \int_{t}^{t+\theta}\left\|g^{\epsilon}\right\|_{H^{-1}(Q)}^{2} \mathrm{~d} s \mathrm{~d} t & \leqslant \sup _{|\theta| \leqslant \delta} \int_{0}^{T}\left(\int_{t}^{t+\theta} \mathrm{d} s\right)^{\frac{1}{2}}\left(\int_{t}^{t+\theta}\left\|g^{\epsilon}\right\|_{H^{-1}(Q)}^{4} \mathrm{~d} s\right)^{\frac{1}{2}} \mathrm{~d} t \\
& \leqslant \delta^{\frac{1}{2}} \int_{0}^{T}\left(\int_{0}^{T}\left\|g^{\epsilon}\right\|_{H^{-1}(Q)}^{4} \mathrm{~d} t\right)^{\frac{1}{2}} \mathrm{~d} t .
\end{aligned}
$$

Now using the assumption made on $g^{\epsilon}$, we have

$$
\mathbb{E} \sup _{0 \leqslant \theta \leqslant \delta} \int_{0}^{T}\left\|\int_{t}^{t+\theta} g^{\epsilon} \mathrm{d} W(s)\right\|_{H^{-1}(Q)}^{2} \mathrm{~d} t \leqslant C(T) \delta^{\frac{1}{2}} .
$$

From (6), (7) and (8), we arrive at

$$
\mathbb{E} \sup _{|\theta| \leqslant \delta} \int_{0}^{T}\left\|u_{t}^{\epsilon}(t+\theta)-u_{t}^{\epsilon}(t)\right\|_{H^{-1}(Q)}^{2} \mathrm{~d} t \leqslant C \delta .
$$

\section{Tightness property of probability measures}

The following lemmas are needed in the proof of the tightness and the study of the properties of the probability measures generated by the sequence $\left(W, u^{\epsilon}, u_{t}^{\epsilon}\right)$.

We have from [49] the following lemma.

Lemma 3. Let $B_{0}, B$ and $B_{1}$ be some Banach spaces such that $B_{0} \subset B \subset B_{1}$ and the injection $B_{0} \subset B$ is compact. For any $1 \leqslant p, q \leqslant \infty$ and $0<s \leqslant 1$ let $E$ be a set bounded in $L^{q}\left(0, T ; B_{0}\right) \cap N^{s, p}(0, T$; $\left.B_{1}\right)$, where

$$
N^{s, p}\left(0, T ; B_{1}\right)=\left\{v \in L^{p}\left(0, T ; B_{1}\right): \sup _{h>0} h^{-s}\|v(t+\theta)-v(t)\|_{L^{p}\left(0, T-\theta, B_{1}\right)}<\infty\right\} .
$$

Then $E$ is relatively compact in $L^{p}(0, T ; B)$.

The following two lemmas are collected from [10]. Let $\mathcal{S}$ be a separable Banach space and consider its Borel $\sigma$-field to be $\mathcal{B}(\mathcal{S})$. We have the following lemmas. 
Lemma 4 (Prokhorov). A sequence of probability measures $\left(\Pi_{n}\right)_{n \in \mathbb{N}}$ on $(\mathcal{S}, \mathcal{B}(\mathcal{S}))$ is tight if and only if it is relatively compact.

Lemma 5 (Skorokhod). Suppose that the probability measures $\left(\mu_{n}\right)_{n \in \mathbb{N}}$ on $(\mathcal{S}, \mathcal{B}(\mathcal{S}))$ weakly converge to a probability measure $\mu$. Then there exist random variables $\xi, \xi_{1}, \ldots, \xi_{n}, \ldots$, defined on a common probability space $(\Omega, \mathcal{F}, \mathbb{P})$, such that $\mathcal{L}\left(\xi_{n}\right)=\mu_{n}$ and $\mathcal{L}(\xi)=\mu$ and

$$
\lim _{n \rightarrow \infty} \xi_{n}=\xi, \quad \mathbb{P} \text {-a.s.; }
$$

the symbol $\mathcal{L}(\cdot)$ stands for the law of $\cdot$

Let us introduce the space $Z=Z_{1} \times Z_{2}$ where

$$
Z_{1}=\left\{\phi: \sup _{0 \leqslant t \leqslant T}\|\phi(t)\|_{H_{0}^{1}(Q)}^{2} \leqslant C_{1}, \sup _{0 \leqslant t \leqslant T}\left\|\phi^{\prime}(t)\right\|_{L^{2}(Q)}^{2} \leqslant C_{1}\right\}
$$

and

$$
Z_{2}=\left\{\psi: \sup _{0 \leqslant t \leqslant T}\|\psi(t)\|_{L^{2}(Q)}^{2} \leqslant C_{3} \text { and } \sup _{n} \frac{1}{\nu_{n}} \sup _{\theta \leqslant \mu_{n}}\left(\int_{0}^{T}\|\psi(t+\theta)-\psi(t)\|_{H^{-1}(Q)}^{2}\right)^{\frac{1}{2}}<\infty\right\} .
$$

We endow $Z$ with the norm

$$
\begin{aligned}
\|(\phi, \psi)\|_{Z}= & \|\phi\|_{Z_{1}}+\|\psi\|_{Z_{2}} \\
= & \sup _{0 \leqslant t \leqslant T}\left\|\phi^{\prime}(t)\right\|_{L^{2}(Q)}+\sup _{0 \leqslant t \leqslant T}\|\phi\|_{H_{0}^{1}(Q)} \\
& +\sup _{0 \leqslant t \leqslant T}\|\psi(t)\|_{L^{2}(Q)}+\sup _{n} \frac{1}{\nu_{n}} \sup _{\theta \leqslant \mu_{n}}\left(\int_{0}^{T}\|\psi(t+\theta)-\psi(t)\|_{H^{-1}(Q)}^{2}\right)^{\frac{1}{2}} .
\end{aligned}
$$

Lemma 6. The above constructed space $Z$ is a compact subset of $L^{2}\left(0, T ; L^{2}(Q)\right) \times L^{2}\left(0, T ; H^{-1}(Q)\right)$.

Proof. Lemma 3 together with a suitable argument due to Bensoussan [8] give the compactness of $Z_{1}$ and $Z_{2}$ in $L^{2}\left(0, T ; L^{2}(Q)\right)$ and $L^{2}\left(0, T ; H^{-1}(Q)\right)$, respectively.

Now consider the space $\mathcal{X}=C\left(0, T ; \mathbb{R}^{m}\right) \times L^{2}\left(0, T ; L^{2}(Q)\right) \times L^{2}\left(0, T ; H^{-1}(Q)\right)$ and $\mathcal{B}(\mathcal{X})$ the $\sigma$ algebra of the Borel sets of $\mathcal{X}$. Let $\Psi_{\epsilon}$ be the $(\mathcal{X}, \mathcal{B}(\mathcal{X}))$-valued measurable map defined on $(\Omega, \mathcal{F}, \mathbb{P})$ by

$$
\Psi_{\epsilon}: \omega \mapsto\left(W(\omega), u^{\epsilon}(\omega), u_{t}^{\epsilon}(\omega)\right) .
$$

Define on $(\mathcal{X}, \mathcal{B}(\mathcal{X}))$ the probability measures $\Pi_{\epsilon}$ by

$$
\Pi_{\epsilon}(A)=\mathbb{P}\left(\Psi_{\epsilon}^{-1}(A)\right) \quad \text { for all } A \in \mathcal{B}(\mathcal{X}) .
$$

Lemma 7. The family of probability measures $\left\{\Pi_{\epsilon}: \epsilon>0\right\}$ is tight in $(\mathcal{X}, \mathcal{B}(\mathcal{X}))$. 
Proof. We carry out the proof following [8,18,38,39] and [44]. For $\delta>0$, we look for compact subsets

$$
W_{\delta} \subset C\left(0, T ; \mathbb{R}^{m}\right), \quad D_{\delta} \subset L^{2}\left(0, T ; L^{2}(Q)\right), \quad E_{\delta} \subset L^{2}\left(0, T ; H^{-1}(Q)\right)
$$

such that

$$
\Pi_{\epsilon}\left\{\left(W, u^{\epsilon}, u_{t}^{\epsilon}\right) \in W_{\delta} \times D_{\delta} \times E_{\delta}\right\} \geqslant 1-\delta .
$$

This is equivalent to

$$
\mathbb{P}\left\{\omega: W(\cdot, \omega) \in W_{\delta}, u^{\epsilon}(\cdot, \omega) \in D_{\delta}, u_{t}^{\epsilon}(\cdot, \omega) \in E_{\delta}\right\} \geqslant 1-\delta,
$$

which can be proved if we can show that

$$
\mathbb{P}\left\{\omega: W(\cdot, \omega) \notin W_{\delta}\right\} \leqslant \delta, \quad \mathbb{P}\left\{u^{\epsilon}(\cdot, \omega) \notin D_{\delta}\right\} \leqslant \delta, \quad \mathbb{P}\left\{u_{t}^{\epsilon}(\cdot, \omega) \notin E_{\delta}\right\} \leqslant \delta .
$$

Let $L_{\delta}$ be a positive constant and $n \in \mathbb{N}$. Then we deal with the set

$$
W_{\delta}=\left\{W(\cdot) \in C\left(0, T ; \mathbb{R}^{m}\right): \sup _{t, s \in[0, T]} n|W(s)-W(t)| \leqslant L_{\delta}:|s-t| \leqslant T n^{-1}\right\} .
$$

Using Arzela's theorem and the fact that $W_{\delta}$ is closed in $C\left(0, T ; \mathbb{R}^{m}\right)$, we ensure the compactness of $W_{\delta}$ in $C\left(0, T ; \mathbb{R}^{m}\right)$. From Markov's inequality

$$
\mathbb{P}(\omega: \eta(\omega) \geqslant \alpha) \leqslant \frac{\mathbb{E}|\eta(\omega)|^{k}}{\alpha^{k}},
$$

where $\eta$ is a nonnegative random variable and $k$ a positive real number, we have

$$
\begin{aligned}
\mathbb{P}\left\{\omega: W(\cdot, \omega) \notin W_{\delta}\right\} & \leqslant \mathbb{P}\left[\bigcup_{n=1}^{\infty}\left(\sup _{t, s \in[0, T]}|W(s)-W(t)| \geqslant \frac{L_{\delta}}{n}:|s-t| \leqslant T n^{-1}\right)\right] \\
& \leqslant \sum_{n=0}^{\infty} \mathbb{P}\left[\bigcup_{j=1}^{n^{6}}\left(\sup _{T j n^{-6} \leqslant t \leqslant T(j+1) n^{-6}}|W(s)-W(t)| \geqslant \frac{L_{\delta}}{n}\right)\right] .
\end{aligned}
$$

But

$$
\mathbb{E}|W(t)-W(s)|^{k} \leqslant(k-1) !(t-s)^{\frac{k}{2}}, \quad k=2,3, \ldots .
$$

For $k=4$, we have

$$
\begin{aligned}
\mathbb{P}\left\{\omega: W(\cdot, \omega) \notin W_{\delta}\right\} & \leqslant \sum_{n=0}^{\infty} \sum_{j=1}^{n^{6}}\left(\frac{n}{L_{\delta}}\right)^{4} \mathbb{E}\left(\sup _{T j n^{-6} \leqslant t \leqslant T(j+1) n^{-6}}\left|W(t)-W\left(j T n^{-6}\right)\right|^{4}\right) \\
& \leqslant C \sum_{n=0}^{\infty} \sum_{j=1}^{n^{6}}\left(\frac{n}{L_{\delta}}\right)^{4}\left(T n^{-6}\right)^{2}=\frac{C T^{2}}{\left(L_{\delta}\right)^{4}} \sum_{n=0}^{\infty} n^{-2} .
\end{aligned}
$$


For the choice $\left(L_{\delta}\right)^{4}=\frac{\left(\sum n^{-2}\right)^{-1}}{3 C T^{2} \delta}$, we have

$$
\mathbb{P}\left\{\omega: W(\cdot, \omega) \notin W_{\delta}\right\} \leqslant \frac{\delta}{3} .
$$

Now, let $K_{\delta}, M_{\delta}$ be positive constants. We define

$$
D_{\delta}=\left\{z: \sup _{0 \leqslant t \leqslant T}\|z(t)\|_{H_{0}^{1}(Q)}^{2} \leqslant K_{\delta}, \sup _{0 \leqslant t \leqslant T}\left\|z^{\prime}(t)\right\|_{L^{2}(Q)}^{2} \leqslant M_{\delta}\right\} .
$$

But Lemma 6 shows that $D_{\delta}$ is compact subset of $L^{2}\left(0, T ; L^{2}(Q)\right)$ for any $\delta>0$. It is easy to see that

$$
\mathbb{P}\left\{u^{\epsilon} \notin D_{\delta}\right\} \leqslant \mathbb{P}\left\{\sup _{0 \leqslant t \leqslant T}\left\|u^{\epsilon}(t)\right\|_{H_{0}^{1}(Q)}^{2} \geqslant K_{\delta}\right\}+\mathbb{P}\left\{\sup _{0 \leqslant t \leqslant T}\left\|u_{t}^{\epsilon}(t)\right\|_{L^{2}(Q)}^{2} \geqslant M_{\delta}\right\} .
$$

Markov's inequality (9) gives

$$
\mathbb{P}\left\{u^{\epsilon} \notin D_{\delta}\right\} \leqslant \frac{1}{K_{\delta}} \mathbb{E} \sup _{0 \leqslant t \leqslant T}\left\|u^{\epsilon}(t)\right\|_{H_{0}^{1}(Q)}^{2}+\frac{1}{M_{\delta}} \mathbb{E} \sup _{0 \leqslant t \leqslant T}\left\|u_{t}^{\epsilon}(t)\right\|_{L^{2}(Q)}^{2} \leqslant \frac{C}{K_{\delta}}+\frac{C}{M_{\delta}}=\frac{\delta}{3}
$$

for $K_{\delta}=M_{\delta}=\frac{6 C}{\delta}$.

Similarly, we let $\mu_{n}, \nu_{m}$ sequences of positive real numbers such that $\mu_{n}, \nu_{n} \rightarrow 0$ as $n \rightarrow \infty$ and define

$$
B_{\delta}=\left\{v: \sup _{0 \leqslant t \leqslant T}\|v(t)\|_{L^{2}(Q)}^{2} \leqslant K_{\delta}^{\prime}, \sup _{\theta \leqslant \mu_{n}} \int_{0}^{T}\|v(t+\theta)-v(t)\|_{H^{-1}(Q)}^{2} \mathrm{~d} t \leqslant \nu_{n} M_{\delta}^{\prime}\right\} .
$$

By Lemma $6 B_{\delta}$ is compact subset of $L^{2}\left(0, T ; H^{-1}(Q)\right)$ for any $\delta>0$. We have

$$
\begin{aligned}
\mathbb{P}\left\{u_{t}^{\epsilon} \notin B_{\delta}\right\} \leqslant & \mathbb{P}\left\{\sup _{0 \leqslant t \leqslant T}\left\|u_{t}^{\epsilon}(t)\right\|_{L^{2}(Q)}^{2} \geqslant K_{\delta}^{\prime}\right\} \\
& +\mathbb{P}\left\{\sup _{\theta \leqslant \mu_{n}} \int_{0}^{T}\left\|u_{t}^{\epsilon}(t+\theta)-u_{t}^{\epsilon}(t)\right\|_{H^{-1}(Q)}^{2} \mathrm{~d} t \geqslant \nu_{n} M_{\delta}^{\prime}\right\} .
\end{aligned}
$$

Again thanks to (9), we obtain

$$
\begin{aligned}
\mathbb{P}\left\{u_{t}^{\epsilon} \notin B_{\delta}\right\} & \leqslant \frac{1}{K_{\delta}^{\prime}} \mathbb{E} \sup _{0 \leqslant t \leqslant T}\left\|u_{t}^{\epsilon}(t)\right\|_{L^{2}(Q)}^{2}+\sum_{n=0}^{\infty} \frac{1}{\nu_{n} M_{\delta}^{\prime}} \mathbb{E}\left\{\sup _{\theta \leqslant \mu_{n}} \int_{0}^{T}\left\|u_{t}^{\epsilon}(t+\theta)-u_{t}^{\epsilon}(t)\right\|_{H^{-1}(Q)}^{2} \mathrm{~d} t\right\} \\
& \leqslant \frac{C}{K_{\delta}^{\prime}}+\frac{C}{M_{\delta}^{\prime}} \sum \frac{\mu_{n}}{\nu_{m}}=\frac{\delta}{3}
\end{aligned}
$$

for $K_{\delta}^{\prime}=\frac{6 C}{\delta}$ and $M_{\delta}^{\prime}=\frac{6 C \sum \frac{\mu_{n}}{\nu_{m}}}{\delta}$. This completes the proof. 
From Lemmas 4 and 7, there exist a subsequence $\left\{\Pi_{\epsilon_{j}}\right\}$ and a measure $\Pi$ such that

$$
\Pi_{\epsilon_{j}} \rightarrow \Pi
$$

weakly. From Lemma 5 , there exist a probability space $(\tilde{\Omega}, \tilde{\mathcal{F}}, \tilde{\mathbb{P}})$ and $\mathcal{X}$-valued random variables $\left(W_{\epsilon_{j}}, u^{\epsilon_{j}}, u_{t}^{\epsilon_{j}}\right),\left(\tilde{W} u, u_{t}\right)$ such that the probability law of $\left(W_{\epsilon_{j}}, u^{\epsilon_{j}}, u_{t}^{\epsilon_{j}}\right)$ is $\Pi_{\epsilon_{j}}$ and that of $\left(\tilde{W} u, u_{t}\right)$ is $\Pi$. Furthermore, we have

$$
\left(W_{\epsilon_{j}}, u^{\epsilon_{j}}, u_{t}^{\epsilon_{j}}\right) \rightarrow\left(\tilde{W}, u, u_{t}\right) \quad \text { in } \mathcal{X}, \tilde{\mathbb{P}} \text {-a.s. }
$$

Let us define the filtration

$$
\tilde{\mathcal{F}}_{t}=\sigma\left\{\tilde{W}(s), u(s), u_{t}(s)\right\}_{0 \leqslant s \leqslant t} .
$$

We show that $\tilde{W}(t)$ is an $\tilde{\mathcal{F}}_{t}$-Wiener process following [8] and [44]. Arguing as in [44] we get that $\left(W_{\epsilon_{j}}, u^{\epsilon_{j}}, u_{t}^{\epsilon_{j}}\right)$ satisfies $\tilde{\mathbb{P}}$-a.s. the problem $\left(P_{\epsilon_{j}}\right)$ in the sense of distributions.

\section{Multiple expansion method}

The goal of multiple expansion method is to assume that the solution $u^{\epsilon}(t, x)$ of the problem $\left(P_{\epsilon}\right)$ depends on the variables $t, x$ as well as the microscale $\frac{x}{\epsilon}$. This means, the solution depends explicitly on the microscale variable $y=\frac{x}{\epsilon}$. Eventually it will be proved that, the solution of the homogenized problem does not depend on the microscale $y=\frac{x}{\epsilon}$.

Let $\phi(t, x, y)(t \in[0, T], x \in Q$ and $y \in Y)$ be a smooth function which is $Y$-periodic. The method of multiple expansion, is to think of the solution $u^{\epsilon}(t, x)$ of the problem $\left(P_{\epsilon}\right)$ is of type $\phi$. Thus we have the following expansions:

$$
\begin{aligned}
& u^{\epsilon}(t, x)=u_{0}\left(t, x, \frac{x}{\epsilon}\right)+\epsilon u_{1}\left(t, x, \frac{x}{\epsilon}\right)+\epsilon^{2} u_{2}\left(t, x, \frac{x}{\epsilon}\right)+\cdots, \\
& f^{\epsilon}(t, x)=f_{0}\left(t, x, \frac{x}{\epsilon}\right)+\epsilon f_{1}\left(t, x, \frac{x}{\epsilon}\right)+\epsilon^{2} f_{2}\left(t, x, \frac{x}{\epsilon}\right)+\cdots, \\
& g^{\epsilon}(t, x)=g_{0}\left(t, x, \frac{x}{\epsilon}\right)+\epsilon g_{1}\left(t, x, \frac{x}{\epsilon}\right)+\epsilon^{2} g_{2}\left(t, x, \frac{x}{\epsilon}\right)+\cdots
\end{aligned}
$$

Since the change of the microscopic scale $y=\frac{x}{\epsilon}$ depends on $\epsilon$, it is clear that $y$ changes faster and faster when $\epsilon$ gets smaller and smaller, compared to the macroscopic scale $x$. Therefore we can think of $x$ and $y$ as being independent variables in the cell problem (microscopic scale level). So if we denote by $\phi^{\epsilon}(t, x)=\phi\left(t, x, \frac{x}{\epsilon}\right)$, we can define the partial derivative of $\phi^{\epsilon}(t, x)$ in $x_{i}, i=1,2, \ldots, n$ as

$$
\frac{\partial \phi^{\epsilon}}{\partial x_{i}}(t, x)=\frac{1}{\epsilon} \frac{\partial \phi}{\partial y_{i}}\left(t, x, \frac{x}{\epsilon}\right)+\frac{\partial \phi}{\partial x_{i}}\left(t, x, \frac{x}{\epsilon}\right), \quad i=1,2, \ldots, n .
$$


Let us define the operator $\mathcal{A}_{\epsilon}:=-\operatorname{div}\left(A_{\epsilon} \nabla\right)$, consequently

$$
\mathcal{A}_{\epsilon} \phi^{\epsilon}(t, x)=\frac{1}{\epsilon^{2}} \mathcal{A}_{0} \phi\left(t, x, \frac{x}{\epsilon}\right)+\frac{1}{\epsilon} \mathcal{A}_{1} \phi\left(t, x, \frac{x}{\epsilon}\right)+\mathcal{A}_{2} \phi\left(t, x, \frac{x}{\epsilon}\right)
$$

where

$$
\begin{aligned}
& \mathcal{A}_{0}:=-\operatorname{div}_{y}\left(A(y) \nabla_{y}\right), \quad \mathcal{A}_{1}:=-\operatorname{div}_{x}\left(A(y) \nabla_{y}\right)-\operatorname{div}_{y}\left(A(y) \nabla_{x}\right), \\
& \mathcal{A}_{2}:=-\operatorname{div}_{x}\left(A(y) \nabla_{x}\right) .
\end{aligned}
$$

Substituting (11)-(13) into the problem $\left(P_{\epsilon}\right)$, we have

$$
\begin{cases}d\left[u_{0 t}+\epsilon u_{1 t}+\epsilon^{2} u_{2 t}+\cdots\right]+\left(\frac{1}{\epsilon^{2}} \mathcal{A}_{0}+\frac{1}{\epsilon} \mathcal{A}_{1}+\mathcal{A}_{2}\right)\left[u_{0}+\epsilon u_{1}+\epsilon^{2} u_{2}+\cdots\right] \mathrm{d} t \\ \quad+\left[f_{0}+\epsilon f_{1}+\epsilon^{2} f_{2}+\cdots\right] \mathrm{d} t+\left[g_{0}+\epsilon g_{1}+\epsilon^{2} g_{2}+\cdots\right] \mathrm{d} W & \text { in } Q \times Y \times(0, T), \\ u^{\epsilon}=0 & \text { on } \partial Q \times(0, T), \\ u^{\epsilon}(x, 0)=0, \quad u_{t}^{\epsilon}(x, 0)=0 & \text { in } Q .\end{cases}
$$

Remark that: The initial condition are taken to be zeros for the sake of simplicity. In fact the initial conditions are irrelevant in obtaining the homogenized problem. Equating to coefficients of equal power terms of $\epsilon$, we obtain the following infinite system of equations

$$
\begin{aligned}
& \left\{\begin{array}{l}
\mathcal{A}_{0} u_{0}=0 \text { in } Y \times(0, T), \\
u_{0} \text { is } Y \text { periodic, }
\end{array}\right. \\
& \left\{\begin{array}{l}
\mathcal{A}_{0} u_{1}=-\mathcal{A}_{1} u_{0} \text { in } Y \times(0, T), \\
u_{1} \text { is } Y \text { periodic, }
\end{array}\right. \\
& \left\{\begin{array}{l}
\mathcal{A}_{0} u_{2} \mathrm{~d} t=\left(f_{0}-\mathcal{A}_{1} u_{1}-\mathcal{A}_{2} u_{0}\right) \mathrm{d} t-\mathrm{d} u_{0 t}+g_{0} \mathrm{~d} W \quad \text { in } Y \times(0, T), \\
u_{2} \text { is } Y \text { periodic, }
\end{array}\right.
\end{aligned}
$$

and

$$
\left\{\begin{array}{l}
\mathcal{A}_{0} u_{k+2} \mathrm{~d} t=\left(f_{k}-\mathcal{A}_{1} u_{k+1}-\mathcal{A}_{2} u_{k}\right) \mathrm{d} t-\mathrm{d} u_{k t}+g_{k} \mathrm{~d} W \quad \text { in } Y \times(0, T), \\
u_{k+2} \text { is } Y \text { periodic, }
\end{array}\right.
$$

for $k \geqslant 1$. Now in order to determine the solution of the problem $\left(P_{\epsilon}\right)$, we need to determine the functions $u_{j}\left(t, x, \frac{x}{\epsilon}\right)$. This can be done successfully by solving the above system in its order i.e.; Start with (14), find the unknown $u_{0}$, use it in Eq. (15) to obtain $u_{1}$ as a function of $u_{0}$ and so on. Notice that the differential operator $\mathcal{A}_{0}$ considered in the above system only acts on the microscopic scale $y$, so the variables $t$ and $x$ are taken as parameters. For the existence and uniqueness of solution of (14) and (15) we refer to [9] and [17]. The following lemma will be very important in our analysis.

Lemma 8. The necessary condition for the above system to have a solution, is that the right-hand sides of Eqs (14)-(17) have zero mean value over $Y$. 
Proof. Since the left-hand side of (14)-(17) is $\mathcal{A}_{0} u_{k}, k=0,1,2, \ldots$ Thus

$$
\begin{aligned}
& \int_{Y} \mathcal{A}_{0} u_{k} \mathrm{~d} y \\
& =-\sum_{i, j=1}^{n} \int_{Y} \frac{\partial}{\partial y_{i}} a_{i, j}(y) \frac{\partial u_{k}(y)}{\partial y_{j}} \mathrm{~d} y \\
& =-\sum_{i, j=1}^{n} \int_{0}^{l_{1}} \int_{0}^{l_{2}} \cdots \int_{0}^{l_{n}} \frac{\partial}{\partial y_{i}} a_{i, j}(y) \frac{\partial u_{k}(y)}{\partial y_{j}} \mathrm{~d} y_{1} \mathrm{~d} y_{2} \cdots \mathrm{d} y_{n} \\
& =-\sum_{i, j=1}^{n} \int_{0}^{l_{1}} \int_{0}^{l_{2}} \cdots \int_{0}^{l_{i-1}} \int_{0}^{l_{i+1}} \cdots \int_{0}^{l_{n}}\left[a_{i, j}\left(l_{i}\right) \frac{\partial u_{k}\left(l_{i}\right)}{\partial y_{j}}\right. \\
& =0 .
\end{aligned}
$$

The last equality is due to the periodicity of $a_{i, j}(y)$ and $u_{k}(t, x, y), k=0,1,2, \ldots$, in $y$, which makes sense only if the right-hand side of Eqs (14)-(17) have zero mean value over $Y$. Thus the proof is complete.

Now let us analyze the solution of (14), since the right-hand side is already equal to zero, we multiply Eq. (14) by $u_{0}$ integrate over $Y$

$$
0=-\int_{Y} \operatorname{div}_{y}\left(A(y) \nabla_{y} u_{0}\right) u_{0} \mathrm{~d} y=\int_{Y} A(y) \nabla_{y} u_{0} \nabla_{y} u_{0} \mathrm{~d} y \geqslant \alpha \int_{Y}\left|\nabla_{y} u_{0}\right|^{2} \mathrm{~d} y .
$$

This is only true if $\nabla_{y} u_{0}=0$ and then $u_{0}$ is independent of $y$, let us write $u_{0}(t, x, y)=u(t, x)$. Therefore we can write (15) as

$$
\operatorname{div}_{y}\left(A(y) \nabla_{y} u_{1}\right)=\nabla_{y} \cdot A(y) \nabla_{x} u(t, x) .
$$

Using the separation of variables we can think of the solution of (18) in the form

$$
u_{1}(t, x, y)=-\chi(y) \cdot \nabla_{x} u(t, x)+\tilde{u}_{1}(t, x),
$$

where $\chi(y)$ is known as the first order corrector, which represents a unique solution to the following PDE

$$
\left\{\begin{array}{l}
\operatorname{div}_{y}\left(A(y) \nabla_{y} \chi(y)\right)=\nabla_{y} \cdot A(y) \text { in } Y, \\
\chi \text { is } Y \text { periodic }
\end{array}\right.
$$

(see e.g. [17, pp. 128-129]). Taking into account (19) and the fact that $u_{0}(t, x, y)=u(t, x)$, we rewrite the right-hand side of (16) as

$$
\left(f_{0}+(A(y)-A(y) \chi(y)) \Delta u+\operatorname{div}_{y}\left(A(y) \nabla_{x}\left[\chi(y) \cdot \nabla_{x} u\right]\right)\right) \mathrm{d} t-\mathrm{d} u_{t}+g_{0} \mathrm{~d} W .
$$


From Lemma 8, we have

$$
\begin{aligned}
\int_{Y} \mathrm{~d} u_{t} \mathrm{~d} y= & \int_{Y}(A(y)-A(y) \chi(y)) \Delta u \mathrm{~d} y \mathrm{~d} t+\int_{Y} \operatorname{div}_{y}\left(A(y) \nabla_{x}\left[\chi(y) \cdot \nabla_{x} u\right]\right) \mathrm{d} y \mathrm{~d} t \\
& +\int_{Y} f_{0} \mathrm{~d} y \mathrm{~d} t+\int_{Y} g_{0} \mathrm{~d} y \mathrm{~d} W
\end{aligned}
$$

or equivalently

$$
\mathrm{d} u_{t}=A_{0} \Delta u \mathrm{~d} t+f(t, x) \mathrm{d} t+g(t, x) \mathrm{d} W,
$$

where $\int_{Y} \operatorname{div}_{y}\left(A(y) \nabla_{x}\left[\chi(y) \cdot \nabla_{x} u\right]\right) \mathrm{d} y \mathrm{~d} t=0, \int_{Y} f_{0}(t, x, y) \mathrm{d} y=f(t, x), \int_{Y} g_{0}(t, x, y) \mathrm{d} y=g(t, x)$ and $A_{0}=\int_{Y}(A(y)-A(y) \chi(y)) \mathrm{d} y$. Notice that (21) with zero initial and boundary conditions is the homogenized problem which has a unique solution due to [35]. As mentioned a while ago one can compute successively the functions of the expansion of the solution $u^{\epsilon}$ in (11). Let us describe $u_{2}$ by substituting (19) into (16). Taking into account (21), an easy computation leads to

$$
\begin{aligned}
\mathcal{A}_{0} u_{2}= & -A_{0} \Delta u+\operatorname{div}_{x}\left(A(y) \nabla_{y} u_{1}\right)+\operatorname{div}_{y}\left(A(y) \nabla_{x} u_{1}\right)+\operatorname{div}_{x}\left(A(y) \nabla_{x} u\right) \\
= & -\sum_{i, j=1}^{n} a_{0 i, j} \frac{\partial^{2} u}{\partial x_{i} \partial x_{j}}+\sum_{i, j=1}^{n} a_{i, j}(y) \frac{\partial^{2} u}{\partial x_{i} \partial x_{j}} \\
& -\sum_{i, j, k=1}^{n} \frac{\partial}{\partial x_{i}}\left(a_{i, j}(y) \frac{\partial \chi_{k}}{\partial y_{j}} \frac{\partial u}{\partial x_{k}}\right)-\sum_{i, j, k=1}^{n} \frac{\partial}{\partial y_{i}}\left(a_{i, j}(y) \chi_{k}(y) \frac{\partial u}{\partial x_{j} \partial x_{k}}\right) .
\end{aligned}
$$

Now renaming the indices, we obtain

$$
-\operatorname{div}_{y}\left(A(y) \nabla_{y} u_{2}\right)=B \Delta u
$$

where

$$
B=-\sum_{i, j=1}^{n} a_{0 i, j}+\sum_{i, j=1}^{n} a_{i, j}(y)-\sum_{i, j, k=1}^{n} a_{i, k}(y) \frac{\partial \chi_{j}(y)}{\partial y_{k}}-\sum_{i, j, k=1}^{n}\left(\frac{a_{k, j}(y) \partial \chi_{i}(y)}{\partial y_{k}}\right) .
$$

Using the separation of variables we can think of the solution of (22) in the form

$$
u_{2}(t, x, y)=\vartheta(y) \Delta_{x} u(t, x)+\tilde{u}_{2}(t, x),
$$

where $\vartheta(y)$ is known as the second order corrector, which represents a unique solution to the following PDE

$$
\left\{\begin{array}{l}
\operatorname{div}_{y}\left(A(y) \nabla_{y} \vartheta(y)\right)=B \quad \text { in } Y, \\
\vartheta \text { is } Y \text { periodic, }
\end{array}\right.
$$


(see e.g. [17, p. 132]). Following similar argument as in [17] we obtain the following error estimate

$$
\mathbb{E}\left\|u^{\epsilon}-\left(u-\chi\left(\frac{x}{\epsilon}\right) \cdot \nabla_{x} u(t, x)+\vartheta\left(\frac{x}{\epsilon}\right) \Delta_{x} u(t, x)\right)\right\|_{H^{1}(Q)} \leqslant C \epsilon^{\frac{1}{2}} .
$$

Having the existence and uniqueness of the cell problems of the correctors from the first and second order, (20) and (23), we can continue to compute and prove the existence and uniqueness of the higher order correctors. Thus, we can compute higher order of the multiple scale expansion

$$
u^{\epsilon}(t, x)=\sum_{i=0}^{\infty} \epsilon^{i} u_{i}\left(t, x, \frac{x}{\epsilon}\right) .
$$

Remark that, in solving the system (14)-(17) all the terms in the right-hand side of the above expansion are in fact functions of the solution $u(t, x)$ of the homogenized problem (i.e. the first term is the solution $u(t, x)$ of the homogenized problem itself, the second term is the product of the gradient of the solution of the homogenized problem and the corrector of the first order. In a similar fashion, the $n$th term is the product of the $(n-1)$ derivative of the solution of the homogenized problem and the corrector of the $(n-1)$ order).

Now, for (24) to be well defined, all higher order derivatives of the solution $u(t, x)$ of the homogenized problem must be in the space $L^{2}\left(\Omega, \mathcal{F}, \mathbb{P} ; C\left([0, T] ; H_{0}^{1}(Q)\right)\right)$ and their respective time derivatives ought to be in the space $L^{2}\left(\Omega, \mathcal{F}, \mathbb{P} ; C\left([0, T] ; L^{2}(Q)\right)\right)$. Further more, in order to prove the error estimate, additional regularity assumptions are needed on the data.

In conclusion, the multiple scale expansion method requires more regularity on the data, though it provides us with more information on the solution of the homogenized problem.

\section{Two scale convergence}

The very well-known div curl lemma was introduced by Murat and Tartar (see e.g. [27] and [52]) to solve the problem of convergence of product of two weakly convergent sequences in the space $L^{2}(Q)$. But this requires extra smoothness to be considered on the sequences, so that one can obtain the limit of the product of two sequences in the sense of distribution. The two scale convergence method is an exceptional approach in handling the assignment of the product of two weakly convergent sequences. Provided that one of the two sequences apart from being bounded in the space $L^{2}(Q)$ satisfies a certain smoothness. This setting for the two scale convergence method has a very unique feature in that, the limit of the sequence depends on additional variable which does not appear in the weak limit. Now let us approach the concept mathematically.

Definition 2. A sequence $\left\{v^{\epsilon}\right\}$ in $L^{p}\left(0, T ; L^{p}(Q)\right)(1<p<\infty)$ is said to be two-scale converge to $v=v(t, x, y), v \in L^{p}\left(0, T ; L^{p}(Q \times Y)\right)$, as $\epsilon \rightarrow 0$ if for any $\psi=\psi(t, x, y) \in L^{p}\left((0, T) \times Q ; C_{\mathrm{per}}^{\infty}(Y)\right)$, one has

$$
\lim _{\epsilon \rightarrow 0} \int_{0}^{T} \int_{Q} v^{\epsilon} \psi^{\epsilon} \mathrm{d} x \mathrm{~d} t=\int_{0}^{T} \int_{Q \times Y} v(t, x, y) \psi(t, x, y) \mathrm{d} y \mathrm{~d} x \mathrm{~d} t
$$

where $\psi^{\epsilon}(t, x)=\psi\left(t, \frac{x}{\epsilon}\right)$, we denote this by $\left\{v^{\epsilon}\right\} \rightarrow v 2$-s in $L^{p}\left(0, T ; L^{p}(Q)\right)$. 
The following lemma is a modification of lemma from [17, Lemma 9.1, p. 174], in which we look at the properties of the test functions we are considering.

Lemma 9. (i) Let $\psi \in L^{p}\left((0, T) \times Q ; C_{\mathrm{per}}(Y)\right), 1<p<\infty$. Then $\psi(\cdot, \cdot, \dot{\bar{\epsilon}}) \in L^{p}\left(0, T ; L^{p}(Q)\right)$ with

$$
\left\|\psi\left(\cdot, \cdot, \frac{\dot{\epsilon}}{\epsilon}\right)\right\|_{L^{p}\left(0, T ; L^{p}(Q)\right)} \leqslant\|\psi(\cdot, \cdot, \cdot)\|_{L^{p}\left((0, T) \times Q ; C_{\mathrm{per}}(Y)\right)}
$$

and

$$
\psi\left(\cdot, \cdot, \frac{\cdot}{\epsilon}\right) \rightarrow \int_{Y} \psi(\cdot, \cdot, y) \mathrm{d} y \quad \text { weakly in } L^{p}\left(0, T ; L^{p}(Q)\right)
$$

(ii) If $\psi(t, x, y)=\psi_{1}(t, x) \psi_{2}(y), \psi_{1} \in L^{p}\left(0, T ; L^{s}(Q)\right), \psi_{2} \in L^{r}(Y), 1 \leqslant s, r<\infty$ such that

$$
\frac{1}{r}+\frac{1}{s}=\frac{1}{p}
$$

Then $\psi\left(\cdot, \cdot, \frac{\dot{\bar{\epsilon}}}{\epsilon}\right) \in L^{p}\left(0, T ; L^{p}(Q)\right)$ and

$$
\psi\left(\cdot, \cdot, \frac{\cdot}{\epsilon}\right) \rightarrow \psi_{1}(\cdot, \cdot) \int_{Y} \psi_{2}(y) \mathrm{d} y \quad \text { weakly in } L^{p}\left(0, T ; L^{p}(Q)\right)
$$

The following theorems are of great importance in obtaining the homogenization result and for their proofs, we refer to [3,17] and [25].

Theorem 2. Let $\left\{u^{\epsilon}\right\}$ be a sequence of functions in $L^{2}\left(0, T ; L^{2}(Q)\right)$ such that

$$
\left\|u^{\epsilon}\right\|_{L^{2}\left(0, T ; L^{2}(Q)\right)}<\infty
$$

Then up to subsequence $u^{\epsilon}$ is two-scale convergent in $L^{2}\left(0, T ; L^{2}(Q)\right)$.

Theorem 3. Let $\left\{u^{\epsilon}\right\}$ be a sequence satisfying the assumptions of Theorem 2. Further more let $\left\{u^{\epsilon}\right\} \subset$ $L^{2}\left(0, T ; H_{0}^{1}(Q)\right)$ such that

$$
\left\|u^{\epsilon}\right\|_{L^{2}\left(0, T ; H_{0}^{1}(Q)\right)}<\infty
$$

Then up to subsequence there exist a couple of functions $\left(u, u_{1}\right)$ with $u \in L^{2}\left(0, T ; H_{0}^{1}(Q)\right)$ and $u_{1} \in$ $L^{2}\left((0, T) \times Q ; H_{\mathrm{per}}(Y)\right)$ such that

$$
\begin{array}{ll}
u^{\epsilon} \rightarrow u & \text { 2-s in } L^{2}\left(0, T ; L^{2}(Q)\right), \\
\nabla u^{\epsilon} \rightarrow \nabla_{x} u+\nabla_{y} u_{1} & \text { 2-s in } L^{2}\left(0, T ; L^{2}(Q)\right) .
\end{array}
$$




\subsection{The homogenization result}

We will now study the asymptotic behavior of the problem $\left(P_{\epsilon_{j}}\right)$, when $\epsilon_{j} \rightarrow 0$ using the two scale convergence method.

Theorem 4. Suppose that the assumptions (A1)-(A5) hold. Let

$$
\begin{array}{ll}
a^{\epsilon_{j}} \rightarrow a & \text { weakly in } H_{0}^{1}(Q), \\
b^{\epsilon_{j}} \rightarrow b & \text { weakly in } L^{2}(Q), \\
f^{\epsilon_{j}} \rightarrow f & \text { weakly in } L^{2}(Q \times(0, T)), \\
g^{\epsilon_{j}} \rightarrow g & \text { weakly in } L^{2}(Q \times(0, T)), \\
g_{t}^{\epsilon_{j}} \rightarrow g_{t} & \text { weakly in } L^{2}(Q \times(0, T)) .
\end{array}
$$

Then there exist a probability space $\left(\tilde{\Omega}, \tilde{\mathcal{F}}, \tilde{\mathbb{P}},\left(\tilde{\mathcal{F}}_{t}\right)_{0 \leqslant t \leqslant T}\right)$ and random variables $\left(u^{\epsilon_{j}}, u_{t}^{\epsilon_{j}}, W_{\epsilon_{j}}\right)$ and $\left(u, u_{t}, \tilde{W}\right)$ such that the convergences $(10)$ and $(31)$ hold. Where $\left(u, u_{t}, \tilde{W}\right)$ satisfies the homogenized problem $(P)$.

Proof. The weak formulation of problem $\left(P_{\epsilon_{j}}\right)$ is

$$
\begin{gathered}
\int_{0}^{T} \int_{Q} \mathrm{~d} u_{t}^{\epsilon_{j}} \Phi(t, x) \mathrm{d} x \mathrm{~d} t+\int_{0}^{T} \int_{Q} A_{\epsilon_{j}} \nabla u^{\epsilon_{j}} \nabla \Phi \mathrm{d} x \mathrm{~d} t \\
=\int_{0}^{T} \int_{Q} f^{\epsilon_{j}} \Phi \mathrm{d} x \mathrm{~d} t+\int_{0}^{T} \int_{Q} g^{\epsilon_{j}} \Phi \mathrm{d} x \mathrm{~d} W_{\epsilon_{j}}
\end{gathered}
$$

for any $\Phi \in D((0, T) \times Q)$. Using estimate (1) and convergence (10) into Theorems 2 and 3, we show the two-scale convergence

$$
\nabla u^{\epsilon_{j}} \rightarrow \nabla_{x} u+\nabla_{y} u_{1} \quad 2-\mathrm{s} \text { in } L^{2}\left(0, T ; L^{p}(Q)\right)
$$

From the estimate (1), we have

$$
u_{t}^{\epsilon_{j}} \rightarrow u_{t} \quad \text { weakly* in } L^{\infty}\left(0, T ; L^{2}(Q)\right) .
$$

Let $\Phi^{\epsilon_{j}}(t, x)=\phi(t, x)+\epsilon_{j} \phi_{1}\left(t, x, \frac{x}{\epsilon_{j}}\right)$ where $\phi \in D((0, T) \times Q)$ and $\phi_{1} \in D\left((0, T) \times Q ; C_{\text {per }}^{\infty}(Y)\right)$. Then we can still consider $\Phi^{\epsilon_{j}}$ as test function in (37). Thus

$$
\begin{aligned}
& -\int_{0}^{T} \int_{Q} u_{t}^{\epsilon_{j}}(t, x)\left[\phi_{t}(t, x)+\epsilon_{j} \phi_{1 t}\left(t, x, \frac{x}{\epsilon_{j}}\right)\right] \mathrm{d} x \mathrm{~d} t \\
& \quad+\int_{0}^{T} \int_{Q} A_{\epsilon_{j}}(x) \nabla u^{\epsilon_{j}}(x, t)\left[\nabla_{x} \phi(t, x)+\epsilon_{j} \nabla_{x} \phi_{1}\left(t, x, \frac{x}{\epsilon_{j}}\right)+\nabla_{y} \phi_{1}\left(t, x, \frac{x}{\epsilon_{j}}\right)\right] \mathrm{d} x \mathrm{~d} t
\end{aligned}
$$




$$
\begin{aligned}
= & \int_{0}^{T} \int_{Q} f^{\epsilon_{j}}(t, x)\left[\phi(t, x)+\epsilon_{j} \phi_{1}\left(t, x, \frac{x}{\epsilon_{j}}\right)\right] \mathrm{d} x \mathrm{~d} t \\
& +\int_{0}^{T} \int_{Q} g^{\epsilon_{j}}(t, x)\left[\phi(t, x)+\epsilon_{j} \phi_{1}\left(t, x, \frac{x}{\epsilon_{j}}\right)\right] \mathrm{d} x \mathrm{~d} W_{\epsilon_{j}} .
\end{aligned}
$$

Let us tackle these terms one by one, when $\epsilon_{j} \rightarrow 0$. Thanks to estimate (26) and convergence (38), we have

$$
\begin{aligned}
\lim _{\epsilon_{j} \rightarrow 0} & \int_{0}^{T} \int_{Q} u_{t}^{\epsilon_{j}}(t, x)\left[\phi_{t}(t, x)+\epsilon_{j} \phi_{1 t}\left(t, x, \frac{x}{\epsilon_{j}}\right)\right] \mathrm{d} x \mathrm{~d} t \\
= & \lim _{\epsilon_{j} \rightarrow 0} \int_{0}^{T} \int_{Q} u_{t}^{\epsilon_{j}}(t, x) \phi_{t}(t, x) \mathrm{d} x \mathrm{~d} t \\
& +\lim _{\epsilon_{j} \rightarrow 0} \epsilon_{j} \int_{0}^{T} \int_{Q} u_{t}^{\epsilon_{j}}(t, x) \phi_{1 t}\left(t, x, \frac{x}{\epsilon_{j}}\right) \mathrm{d} x \mathrm{~d} t \\
= & \int_{0}^{T} \int_{Q} u_{t}(t, x) \phi_{t}(t, x) \mathrm{d} x \mathrm{~d} t, \quad \tilde{\mathbb{P}} \text {-a.s. }
\end{aligned}
$$

The second term can be written as follows

$$
\begin{gathered}
\lim _{\epsilon_{j} \rightarrow 0} \int_{0}^{T} \int_{Q} \nabla u^{\epsilon_{j}}(x, t) A_{\epsilon_{j}}\left[\nabla_{x} \phi(t, x)+\nabla_{y} \phi_{1}\left(t, x, \frac{x}{\epsilon_{j}}\right)\right] \mathrm{d} x \mathrm{~d} t \\
\quad+\lim _{\epsilon_{j} \rightarrow 0} \epsilon_{j} \int_{0}^{T} \int_{Q} A_{\epsilon_{j}} \nabla u^{\epsilon_{j}}(x, t) \nabla_{x} \phi_{1}\left(t, x, \frac{x}{\epsilon_{j}}\right) \mathrm{d} x \mathrm{~d} t
\end{gathered}
$$

since $A_{\epsilon_{j}} \in L^{\infty}(Y)$ and $\nabla_{x} \phi(t, x)+\nabla_{y} \phi_{1}(t, x, y) \in L_{\text {per }}^{2}(Y ; C(Q \times(0, T)))$, we regard $A_{\epsilon_{j}}\left[\nabla_{x} \phi(t, x)+\right.$ $\left.\nabla_{y} \phi_{1}\left(t, x, \frac{x}{\epsilon_{j}}\right)\right]$ as test function in the two-scale convergence of the gradient in the first term in (40). Therefore

$$
\begin{aligned}
& \lim _{\epsilon_{j} \rightarrow 0} \int_{0}^{T} \int_{Q} \nabla u^{\epsilon_{j}}(x, t) A_{\epsilon_{j}}\left[\nabla_{x} \phi(t, x)+\nabla_{y} \phi_{1}\left(t, x, \frac{x}{\epsilon_{j}}\right)\right] \mathrm{d} x \mathrm{~d} t \\
& \quad=\int_{0}^{T} \int_{Q \times Y} A(y)\left[\nabla_{x} u(t, x)+\nabla_{y} u_{1}(t, x, y)\right]\left[\nabla_{x} \phi(t, x)+\nabla_{y} \phi_{1}(t, x, y)\right] \mathrm{d} y \mathrm{~d} x \mathrm{~d} t .
\end{aligned}
$$

Thanks to Hölder inequality, (26) and the fact that $A_{\epsilon_{j}} \nabla u^{\epsilon_{j}}$ is bounded in $L^{\infty}\left(0, T ; L^{2}(Q)\right)$, we have

$$
\lim _{\epsilon_{j} \rightarrow 0} \epsilon_{j} \int_{0}^{T} \int_{Q} A_{\epsilon_{j}} \nabla u^{\epsilon_{j}}(x, t) \nabla_{x} \phi_{1}\left(t, x, \frac{x}{\epsilon_{j}}\right) \mathrm{d} x \mathrm{~d} t=0, \quad \tilde{\mathbb{P}} \text {-a.s. }
$$


Thanks to estimate (26) and convergence (34), we have

$$
\begin{aligned}
& \lim _{\epsilon_{j} \rightarrow 0} \int_{0}^{T} \int_{Q} f^{\epsilon_{j}}(t, x)\left[\phi(t, x)+\epsilon_{j} \phi_{1}\left(t, x, \frac{x}{\epsilon_{j}}\right)\right] \mathrm{d} x \mathrm{~d} t \\
& =\lim _{\epsilon_{j} \rightarrow 0} \int_{0}^{T} \int_{Q} f^{\epsilon_{j}}(t, x) \phi(t, x) \mathrm{d} x \mathrm{~d} t+\lim _{\epsilon_{j} \rightarrow 0} \epsilon_{j} \int_{0}^{T} \int_{Q} f^{\epsilon_{j}}(t, x) \phi_{1}\left(t, x, \frac{x}{\epsilon_{j}}\right) \mathrm{d} x \mathrm{~d} t \\
& =\int_{0}^{T} \int_{Q} f(t, x) \phi(t, x) \mathrm{d} x \mathrm{~d} t .
\end{aligned}
$$

In the following we show that

$$
\lim _{\epsilon_{j} \rightarrow 0} \int_{0}^{T} \int_{Q} g^{\epsilon_{j}}(t, x) \phi(t, x) \mathrm{d} x \mathrm{~d} W_{\epsilon_{j}}=\int_{0}^{T} \int_{Q} g(t, x) \phi(t, x) \mathrm{d} x \mathrm{~d} \tilde{W}, \quad \tilde{\mathbb{P}}-\text { a.s. }
$$

Using integration by parts, we have

$$
\begin{aligned}
\lim _{\epsilon_{j} \rightarrow 0} & \int_{0}^{T} \int_{Q} g^{\epsilon_{j}}(t, x) \phi(t, x) \mathrm{d} x \mathrm{~d} W_{\epsilon_{j}} \\
= & \lim _{\epsilon_{j} \rightarrow 0}\left[\left.W_{\epsilon_{j}} \int_{Q} g(t, x) \phi(t, x) \mathrm{d} x\right|_{0} ^{T}-\int_{0}^{T} \int_{Q} g_{t}^{\epsilon_{j}}(t, x) \phi(t, x) W_{\epsilon_{j}} x \mathrm{~d} t\right. \\
& \left.-\int_{0}^{T} \int_{Q} g^{\epsilon_{j}}(t, x) \phi_{t}(t, x) W_{\epsilon_{j}} \mathrm{~d} x \mathrm{~d} t\right] .
\end{aligned}
$$

In the first term in the right-hand side of (41), we pass to the limit using the strong convergence (10), to obtain

$$
\left.\lim _{\epsilon_{j} \rightarrow 0} W_{\epsilon_{j}} \int_{Q} g(t, x) \phi(t, x) \mathrm{d} x\right|_{0} ^{T}=\left.\tilde{W} \int_{Q} g(t, x) \phi(t, x) \mathrm{d} x\right|_{0} ^{T}, \quad \tilde{\mathbb{P}} \text {-a.s., }
$$

the second term can be written as

$$
\lim _{\epsilon_{j} \rightarrow 0} \int_{0}^{T} \int_{Q} g_{t}^{\epsilon_{j}}(t, x) \phi(t, x)\left(W_{\epsilon_{j}}-\tilde{W}\right) \mathrm{d} x \mathrm{~d} t+\lim _{\epsilon_{j} \rightarrow 0} \int_{0}^{T} \int_{Q} g_{t}^{\epsilon_{j}}(t, x) \phi(t, x) \tilde{W} \mathrm{~d} x \mathrm{~d} t,
$$

the first term of (42) will converge to zero using (10) and the assumptions on $g^{\epsilon_{j}}$ and $\phi$

$$
\begin{aligned}
& \lim _{\epsilon_{j} \rightarrow 0}\left|\int_{0}^{T} \int_{Q} g_{t}^{\epsilon_{j}}(t, x) \phi(t, x)\left(W_{\epsilon_{j}}-\tilde{W}\right) \mathrm{d} x \mathrm{~d} t\right| \\
& \quad \leqslant \lim _{\epsilon_{j} \rightarrow 0} \sup _{t \in[0, T]}\left|W_{\epsilon_{j}}-\tilde{W}\right| \int_{0}^{T} \int_{Q}\left|g_{t}^{\epsilon_{j}}(t, x) \phi(t, x)\right| \mathrm{d} x \mathrm{~d} t \leqslant \lim _{\epsilon j \rightarrow 0} C \sup _{t \in[0, T]}\left|W_{\epsilon_{j}}-\tilde{W}\right|=0, \quad \tilde{\mathbb{P}} \text {-a.s. }
\end{aligned}
$$


Thanks to the weak convergence (36), we show that

$$
\lim _{\epsilon_{j} \rightarrow 0} \int_{0}^{T} \int_{Q} g_{t}^{\epsilon_{j}}(t, x) \phi(t, x) \tilde{W} \mathrm{~d} x \mathrm{~d} t=\int_{0}^{T} \int_{Q} g_{t} \phi(t, x) \tilde{W} \mathrm{~d} x \mathrm{~d} t .
$$

Similarly, we treat the last term in the right-hand side of (41)

$$
\lim _{\epsilon_{j} \rightarrow 0} \int_{0}^{T} \int_{Q} g^{\epsilon_{j}}(t, x) \phi_{t}(t, x)\left(W_{\epsilon_{j}}-\tilde{W}\right) \mathrm{d} x \mathrm{~d} t+\lim _{\epsilon_{j} \rightarrow 0} \int_{0}^{T} \int_{Q} g^{\epsilon_{j}}(t, x) \phi_{t}(t, x) \tilde{W} \mathrm{~d} x \mathrm{~d} t .
$$

The first term of (44) will converge to zero and thanks to (35), we have

$$
\lim _{\epsilon_{j} \rightarrow 0} \int_{0}^{T} \int_{Q} g^{\epsilon_{j}}(t, x) \phi_{t}(t, x) \tilde{W} \mathrm{~d} x \mathrm{~d} t=\int_{0}^{T} \int_{Q} g(t, x) \phi_{t}(t, x) \tilde{W} \mathrm{~d} x \mathrm{~d} t .
$$

Now we want to show that

$$
\lim _{\epsilon_{j} \rightarrow 0} \epsilon_{j} \int_{0}^{T} \int_{Q} g^{\epsilon_{j}}(t, x) \phi_{1}\left(t, x, \frac{x}{\epsilon_{j}}\right) \mathrm{d} x \mathrm{~d} W_{\epsilon_{j}}=0, \quad \tilde{\mathbb{P}} \text {-a.s. }
$$

Thanks to Burkhölder-Davis-Gundy's inequality, the assumptions on $g^{\epsilon_{j}}$ and (26), we have

$$
\begin{aligned}
& \lim _{\epsilon_{j} \rightarrow 0} \epsilon_{j} \tilde{\mathbb{E}} \sup _{0 \in[0, T]}\left|\int_{0}^{T} \int_{Q} g^{\epsilon_{j}}(t, x) \phi_{1}\left(t, x, \frac{x}{\epsilon_{j}}\right) \mathrm{d} x \mathrm{~d} W_{\epsilon_{j}}\right| \\
& \leqslant C \lim _{\epsilon_{j} \rightarrow 0} \epsilon_{j} \tilde{\mathbb{E}}\left(\int_{0}^{T}\left(\int_{Q} g^{\epsilon_{j}}(t, x) \phi_{1}\left(t, x, \frac{x}{\epsilon_{j}}\right) \mathrm{d} x\right)^{2} \mathrm{~d} t\right)^{\frac{1}{2}} \\
& \leqslant C_{1} \lim _{\epsilon_{j} \rightarrow 0} \epsilon_{j} \tilde{\mathbb{E}}\left(\int_{0}^{T}\left\|g^{\epsilon_{j}}\right\|_{L^{2}(Q)}\left\|\phi_{1}\left(t, x, \frac{x}{\epsilon_{j}}\right)\right\|_{L^{2}(Q)} \mathrm{d} t\right)^{\frac{1}{2}} \\
& \leqslant C_{1} \lim _{\epsilon_{j} \rightarrow 0} \epsilon_{j}\left(\int_{0}^{T}\left\|g^{\epsilon_{j}}\right\|_{L^{2}(Q)} \mathrm{d} t\right)^{\frac{1}{2}} \rightarrow 0, \quad \tilde{\mathbb{P}}_{- \text {a.s. }}
\end{aligned}
$$

Combining the above convergences, we obtain

$$
\begin{aligned}
& -\int_{0}^{T} \int_{Q} u_{t}(t, x) \phi_{t}(t, x) \mathrm{d} x \mathrm{~d} t \\
& \quad+\int_{0}^{T} \int_{Q \times Y} A(y)\left[\nabla_{x} u(t, x)+\nabla_{y} u_{1}(t, x, y)\right]\left[\nabla_{x} \phi(t, x)+\nabla_{y} \phi_{1}(t, x, y)\right] \mathrm{d} y \mathrm{~d} x \mathrm{~d} t \\
& =\int_{0}^{T} \int_{Q} f(t, x) \phi(t, x) \mathrm{d} x \mathrm{~d} t+\int_{0}^{T} \int_{Q} g(t, x) \phi(t, x) \tilde{W} \mathrm{~d} x \mathrm{~d} t .
\end{aligned}
$$


Choosing in the first stage $\phi=0$ and after $\phi_{1}=0$, the problem (46) is equivalent to the following system of integral equations

$$
\int_{0}^{T} \int_{Q \times Y} A(y)\left[\nabla_{x} u(t, x)+\nabla_{y} u_{1}(t, x, y)\right]\left[\nabla_{y} \phi_{1}(t, x, y)\right] \mathrm{d} y \mathrm{~d} x \mathrm{~d} t=0
$$

and

$$
\begin{aligned}
& -\int_{0}^{T} \int_{Q} u_{t}(t, x) \phi_{t}(t, x) \mathrm{d} x \mathrm{~d} t \\
& \quad+\int_{0}^{T} \int_{Q \times Y} A(y)\left[\nabla_{x} u(t, x)+\nabla_{y} u_{1}(t, x, y)\right]\left[\nabla_{x} \phi(t, x)\right] \mathrm{d} y \mathrm{~d} x \mathrm{~d} t \\
& =\int_{0}^{T} \int_{Q} f(t, x) \phi(t, x) \mathrm{d} x \mathrm{~d} t+\int_{0}^{T} \int_{Q} g(t, x) \phi(t, x) \mathrm{d} \tilde{W} \mathrm{~d} x .
\end{aligned}
$$

Equation (47), is nothing else but the weak formulation of Eq. (15) which has a unique solution given by (19) in terms of $u$. As for the uniqueness of the solution of (48), we prove it as follows. Using (19) into (48), one obtains that (48) is the weak formulation of the equation

$$
\mathrm{d} u_{t}=A_{0} \Delta u \mathrm{~d} t+f(t, x) \mathrm{d} t+g(t, x) \tilde{W},
$$

where

$$
A_{0}=\int_{Y}\left(A(y)-A(y) \nabla_{y} \chi(y)\right) \mathrm{d} y .
$$

But the initial boundary value problem corresponding to (49) has a unique solution by [35].

It remains to show that $u(x, 0)=a(x)$ and $u_{t}(x, 0)=b(x)$. Notice that Eq. (37) is valid for $\Phi^{\epsilon_{j}}(t, x)=$ $\phi(t, x)+\epsilon_{j} \phi_{1}\left(t, x, \frac{x}{\epsilon_{j}}\right)$ where $\phi \in C^{\infty}((0, T) \times Q)$ and $\phi_{1} \in D\left((0, T) \times Q ; C_{\mathrm{per}}^{\infty}(Y)\right)$, such that $\phi(0, x)=$ $v(x)$ and $\phi(T, x)=0$. Now integrating the first term in (37) by parts, we obtain

$$
\begin{aligned}
& -\int_{0}^{T} \int_{Q} u_{t}^{\epsilon_{j}}(t, x)\left[\phi_{t}(t, x)+\epsilon_{j} \phi_{1 t}\left(t, x, \frac{x}{\epsilon_{j}}\right)\right] \mathrm{d} x \mathrm{~d} t \\
& \quad+\int_{0}^{T} \int_{Q} A_{\epsilon_{j}}(x) \nabla u^{\epsilon_{j}}(x, t)\left[\nabla_{x} \phi(t, x)+\epsilon_{j} \nabla_{x} \phi_{1}\left(t, x, \frac{x}{\epsilon_{j}}\right)+\nabla_{y} \phi_{1}\left(t, x, \frac{x}{\epsilon_{j}}\right)\right] \mathrm{d} x \mathrm{~d} t \\
& =\int_{0}^{T} \int_{Q} f^{\epsilon_{j}}(t, x)\left[\phi(t, x)+\epsilon_{j} \phi_{1}\left(t, x, \frac{x}{\epsilon_{j}}\right)\right] \mathrm{d} x \mathrm{~d} t \\
& \quad+\int_{0}^{T} \int_{Q} g^{\epsilon_{j}}(t, x)\left[\phi(t, x)+\epsilon_{j} \phi_{1}\left(t, x, \frac{x}{\epsilon_{j}}\right)\right] \mathrm{d} x \mathrm{~d} W_{\epsilon_{j}} \\
& \quad+\int_{Q} u_{t}^{\epsilon_{j}}(x, 0) v(x) \mathrm{d} x
\end{aligned}
$$


where we pass to the limit, to get

$$
\begin{aligned}
& -\int_{0}^{T} \int_{Q} u_{t}(t, x) \phi_{t}(t, x) \mathrm{d} x \mathrm{~d} t \\
& \quad+\int_{0}^{T} \int_{Q \times Y} A(y)\left[\nabla_{x} u(t, x)+\nabla_{y} u_{1}(t, x, y)\right]\left[\nabla_{x} \phi(t, x)+\nabla_{y} \phi_{1}(t, x, y)\right] \mathrm{d} y \mathrm{~d} x \mathrm{~d} t \\
& =\int_{0}^{T} \int_{Q} f(t, x) \phi(t, x) \mathrm{d} x \mathrm{~d} t+\int_{0}^{T} \int_{Q} g(t, x) \phi(t, x) \tilde{W} \mathrm{~d} x \mathrm{~d} t+\int_{Q} b(x) v(x) \mathrm{d} x .
\end{aligned}
$$

The integration by parts, in the first term gives

$$
\begin{aligned}
\int_{0}^{T} \int_{Q} \mathrm{~d} u_{t}(t, x) \phi(t, x) \mathrm{d} x+\int_{Q} u_{t}(x, 0) v(x) \mathrm{d} x \\
\quad+\int_{0}^{T} \int_{Q \times Y} A(y)\left[\nabla_{x} u(t, x)+\nabla_{y} u_{1}(t, x, y)\right]\left[\nabla_{x} \phi(t, x)+\nabla_{y} \phi_{1}(t, x, y)\right] \mathrm{d} y \mathrm{~d} x \mathrm{~d} t \\
=\int_{0}^{T} \int_{Q} f(t, x) \phi(t, x) \mathrm{d} x \mathrm{~d} t+\int_{0}^{T} \int_{Q} g(t, x) \phi(t, x) \tilde{W} \mathrm{~d} x \mathrm{~d} t+\int_{Q} b(x) v(x) \mathrm{d} x .
\end{aligned}
$$

Since Eq. (46) still also valid for $\phi \in C^{\infty}((0, T) \times Q)$, we deduce that

$$
\int_{Q} u_{t}(x, 0) v(x) \mathrm{d} x=\int_{Q} b(x) v(x) \mathrm{d} x
$$

for any $v \in C^{\infty}(Q)$, which implies that $u_{t}(x, 0)=b(x)$. For the other initial condition, we regard $\Phi^{\epsilon_{j}}(t, x)=\phi(t, x)+\epsilon_{j} \phi_{1}\left(t, x, \frac{x}{\epsilon_{j}}\right)$ where $\phi \in C^{\infty}((0, T) \times Q)$ and $\phi_{1} \in D\left((0, T) \times Q ; C_{\mathrm{per}}^{\infty}(Y)\right)$, such that $\phi(0, x)=0, \phi_{t}(0, x)=v(x)$ and $\phi(T, x)=0=\phi_{t}(T, x)$ as a test function in (37). The integration by parts twice in the first term of (37) gives

$$
\begin{aligned}
& \int_{0}^{T} \int_{Q} u_{t}^{\epsilon_{j}}(t, x)\left[\phi_{t t}(t, x)+\epsilon_{j} \phi_{1 t t}\left(t, x, \frac{x}{\epsilon_{j}}\right)\right] \mathrm{d} x \mathrm{~d} t \\
& \quad+\int_{0}^{T} \int_{Q} A_{\epsilon_{j}}(x) \nabla u^{\epsilon_{j}}(x, t)\left[\nabla_{x} \phi(t, x)+\epsilon_{j} \nabla_{x} \phi_{1}\left(t, x, \frac{x}{\epsilon_{j}}\right)+\nabla_{y} \phi_{1}\left(t, x, \frac{x}{\epsilon_{j}}\right)\right] \mathrm{d} x \mathrm{~d} t \\
& =\int_{0}^{T} \int_{Q} f^{\epsilon_{j}}(t, x)\left[\phi(t, x)+\epsilon_{j} \phi_{1}\left(t, x, \frac{x}{\epsilon_{j}}\right)\right] \mathrm{d} x \mathrm{~d} t \\
& \quad+\int_{0}^{T} \int_{Q} g^{\epsilon_{j}}(t, x)\left[\phi(t, x)+\epsilon_{j} \phi_{1}\left(t, x, \frac{x}{\epsilon_{j}}\right)\right] \mathrm{d} x \mathrm{~d} W_{\epsilon_{j}} \\
& \quad-\int_{Q} u^{\epsilon_{j}}(x, 0) v(x) \mathrm{d} x
\end{aligned}
$$


where we pass to the limit, we obtain

$$
\begin{aligned}
& \int_{0}^{T} \int_{Q} u_{t}(t, x) \phi_{t t}(t, x) \mathrm{d} x \mathrm{~d} t \\
& \quad+\int_{0}^{T} \int_{Q \times Y} A(y)\left[\nabla_{x} u(t, x)+\nabla_{y} u_{1}(t, x, y)\right]\left[\nabla_{x} \phi(t, x)+\nabla_{y} \phi_{1}(t, x, y)\right] \mathrm{d} y \mathrm{~d} x \mathrm{~d} t \\
& =\int_{0}^{T} \int_{Q} f(t, x) \phi(t, x) \mathrm{d} x \mathrm{~d} t+\int_{0}^{T} \int_{Q} g(t, x) \phi(t, x) \tilde{W} \mathrm{~d} x \mathrm{~d} t-\int_{Q} a(x) v(x) \mathrm{d} x .
\end{aligned}
$$

We integrate by parts again to obtain

$$
\begin{aligned}
& -\int_{0}^{T} \int_{Q} u_{t}(t, x) \phi_{t}(t, x) \mathrm{d} x \mathrm{~d} t-\int_{Q} u(x, 0) v(x) \mathrm{d} x \\
& \quad+\int_{0}^{T} \int_{Q \times Y} A(y)\left[\nabla_{x} u(t, x)+\nabla_{y} u_{1}(t, x, y)\right]\left[\nabla_{x} \phi(t, x)+\nabla_{y} \phi_{1}(t, x, y)\right] \mathrm{d} y \mathrm{~d} x \mathrm{~d} t \\
& =\int_{0}^{T} \int_{Q} f(t, x) \phi(t, x) \mathrm{d} x \mathrm{~d} t+\int_{0}^{T} \int_{Q} g(t, x) \phi(t, x) \tilde{W} \mathrm{~d} x \mathrm{~d} t-\int_{Q} a(x) v(x) \mathrm{d} x .
\end{aligned}
$$

Using the same argument as before, we obtain that $u(x, 0)=a(x)$. Thus the proof is complete.

We note the triple $\left(\tilde{W}, u, u_{t}\right)$ is a probabilistic weak solution of $(P)$ which is unique. Thus by the infinite dimensional version of Yamada-Watanabe's theorem (see [32]), we get that $\left(W, u, u_{t}\right)$ is unique strong solution of $(P)$. Thus up to distribution (probability law) the whole sequence of solutions of $\left(P_{\epsilon}\right)$ converges to the solution of problem $(P)$.

\subsection{The corrector result}

Theorem 5. Let the assumptions of Theorem 4 be fulfilled. Assume that $\nabla_{y} \chi(y) \in\left[L^{r}(Y)\right]^{n}$ and $\nabla u \in$ $L^{2}\left(0, T ;\left[L^{s}(Y)\right]^{n}\right)$ with $1 \leqslant r, s<\infty$ such that

$$
\frac{1}{r}+\frac{1}{s}=\frac{1}{2}
$$

Furthermore, let

$$
\begin{array}{ll}
-\operatorname{div}\left(A_{\epsilon_{j}} \nabla a^{\epsilon_{j}}\right) \rightarrow-\operatorname{div}\left(A_{0} \nabla a\right) & \text { strongly in } H^{-1}(Q), \\
b^{\epsilon_{j}} \rightarrow b & \text { strongly in } L^{2}(Q), \\
f^{\epsilon_{j}} \rightarrow f & \text { strongly in } L^{2}(Q \times(0, T)), \\
g^{\epsilon_{j}} \rightarrow g & \text { strongly in } L^{2}(Q \times(0, T)) .
\end{array}
$$


Then

$$
\begin{aligned}
& u_{t}^{\epsilon_{j}}-u_{t}-\epsilon_{j} u_{1 t}\left(\cdot, \cdot, \frac{\cdot}{\epsilon_{j}}\right) \rightarrow 0 \quad \text { strongly in } L^{2}\left(0, T ; H^{-1}(Q)\right), \tilde{\mathbb{P}} \text {-a.s. } \\
& u^{\epsilon_{j}}-u-\epsilon_{j} u_{1}\left(\cdot, \cdot, \frac{\cdot}{\epsilon_{j}}\right) \rightarrow 0 \quad \text { strongly in } L^{2}\left(0, T ; H^{1}(Q)\right), \tilde{\mathbb{P}} \text {-a.s. }
\end{aligned}
$$

Proof. It is easy to see that

$$
\lim _{\epsilon_{j} \rightarrow 0} \epsilon_{j} u_{1 t}\left(\cdot, \cdot, \frac{\cdot}{\epsilon_{j}}\right) \rightarrow 0 \quad \text { in } L^{2}\left(0, T ; L^{2}(Q)\right), \tilde{\mathbb{P}} \text {-a.s. }
$$

Then from the compact embedding $L^{2}(Q) \subset \subset H^{-1}(Q)$ and the convergence (10) we have

$$
u_{t}^{\epsilon_{j}}-u_{t}-\epsilon_{j} u_{1 t}\left(\cdot, \cdot, \frac{\cdot}{\epsilon_{j}}\right) \rightarrow 0 \quad \text { in } L^{2}\left(0, T ; H^{-1}(Q)\right), \tilde{\mathbb{P}} \text {-a.s. }
$$

Thus (55) holds. Similarly we show that

$$
u^{\epsilon_{j}}-u-\epsilon_{j} u_{1}\left(\cdot, \cdot, \frac{\cdot}{\epsilon_{j}}\right) \rightarrow 0 \quad \text { strongly in } L^{2}\left(0, T ; L^{2}(Q)\right), \tilde{\mathbb{P}} \text {-a.s. }
$$

It remains to show that

$$
\nabla\left(u^{\epsilon_{j}}-u-\epsilon_{j} u_{1}\left(\cdot, \cdot, \frac{\cdot}{\epsilon_{j}}\right)\right) \rightarrow 0 \quad \text { strongly in } L^{2}\left(0, T ;\left[L^{2}(Q)\right]^{n}\right), \tilde{\mathbb{P}}-\text { a.s. }
$$

First

$$
\nabla\left(u^{\epsilon_{j}}-u-\epsilon_{j} u_{1}\left(\cdot, \cdot, \frac{\cdot}{\epsilon_{j}}\right)\right)=\nabla u^{\epsilon_{j}}-\nabla u-\nabla_{y} u_{1}\left(\cdot, \cdot, \frac{\dot{\bar{c}}}{\epsilon_{j}}\right)-\epsilon_{j} \nabla u_{1}\left(\cdot, \cdot, \frac{\dot{\bar{\epsilon}}}{\epsilon_{j}}\right) .
$$

Again

$$
\lim _{\epsilon_{j} \rightarrow 0} \epsilon_{j} \nabla u_{1}\left(\cdot, \cdot, \frac{\cdot}{\epsilon_{j}}\right) \rightarrow 0 \quad \text { in } L^{2}\left(0, T ;\left[L^{2}(Q)\right]^{n}\right), \tilde{\mathbb{P}} \text {-a.s. }
$$

Now from the ellipticity assumption on the matrix $A$, we have

$$
\begin{aligned}
\alpha \mathbb{E} \int_{0}^{T} & \left\|\nabla u^{\epsilon_{j}}-\nabla u-\nabla_{y} u_{1}\left(\cdot, \cdot, \frac{\cdot}{\epsilon_{j}}\right)\right\|_{L^{2}(Q)}^{2} \mathrm{~d} t \\
\leqslant & \mathbb{E} \int_{0}^{T} \int_{Q} A\left(\frac{x}{\epsilon_{j}}\right)\left(\nabla u^{\epsilon_{j}}-\nabla u-\nabla_{y} u_{1}\left(\cdot, \cdot, \frac{\cdot}{\epsilon_{j}}\right)\right) \\
& \cdot\left(\nabla u^{\epsilon_{j}}-\nabla u-\nabla_{y} u_{1}\left(\cdot, \cdot, \frac{\cdot}{\epsilon_{j}}\right)\right) \mathrm{d} x \mathrm{~d} t
\end{aligned}
$$




$$
\begin{aligned}
= & \mathbb{E} \int_{0}^{T} \int_{Q} A_{\epsilon_{j}} \nabla u^{\epsilon_{j}} \nabla u^{\epsilon_{j}} \mathrm{~d} x \mathrm{~d} t \\
& -2 \mathbb{E} \int_{0}^{T} \int_{Q} \nabla u^{\epsilon_{j}} A\left(\frac{x}{\epsilon_{j}}\right)\left(\nabla u+\nabla_{y} u_{1}\left(\cdot, \cdot, \frac{\cdot}{\epsilon_{j}}\right)\right) \mathrm{d} x \mathrm{~d} t \\
& +\mathbb{E} \int_{0}^{T} \int_{Q} A\left(\frac{x}{\epsilon_{j}}\right)\left(\nabla u+\nabla_{y} u_{1}\left(\cdot, \cdot, \frac{\cdot}{\epsilon_{j}}\right)\right) \cdot\left(\nabla u+\nabla_{y} u_{1}\left(\cdot, \cdot, \frac{\cdot}{\epsilon_{j}}\right)\right) \mathrm{d} x \mathrm{~d} t .
\end{aligned}
$$

Let us pass to the limit in this inequality. We start with

$$
\mathbb{E} \int_{Q} A_{\epsilon_{j}} \nabla u^{\epsilon_{j}} \nabla u^{\epsilon_{j}} \mathrm{~d} x
$$

Applying Itô's formula to $\left\|u_{t}^{\epsilon_{j}}\right\|_{L^{2}(Q)}^{2}$, using problem $\left(P_{\epsilon_{j}}\right)$ and the symmetry of $A_{\epsilon_{j}}$ and integrating over $(0, t)$, we obtain

$$
\begin{aligned}
& \left\|u_{t}^{\epsilon_{j}}\right\|_{L^{2}(Q)}^{2}+\int_{Q} A_{\epsilon_{j}} \nabla u^{\epsilon_{j}} \nabla u^{\epsilon_{j}} \mathrm{~d} x \\
& =\left\|b^{\epsilon_{j}}\right\|_{L^{2}(Q)}^{2}+\int_{Q} A_{\epsilon_{j}} \nabla a^{\epsilon_{j}} \nabla a^{\epsilon_{j}} \mathrm{~d} x \\
& \quad+2 \int_{0}^{t}\left(f^{\epsilon_{j}}, u_{t}^{\epsilon_{j}}\right) \mathrm{d} s+2 \int_{0}^{t}\left(g^{\epsilon_{j}}, u_{t}^{\epsilon_{j}}\right) \mathrm{d} W_{\epsilon_{j}}+\int_{0}^{t}\left\|g^{\epsilon_{j}}\right\|_{L^{2}(Q)}^{2} .
\end{aligned}
$$

Taking the expectation in both sides of the above equation, we get

$$
\begin{aligned}
\lim _{\epsilon_{j} \rightarrow 0}\left[\mathbb{E}\left\|u_{t}^{\epsilon_{j}}\right\|_{L^{2}(Q)}^{2}+\mathbb{E} \int_{Q} A_{\epsilon_{j}} \nabla u^{\epsilon_{j}} \nabla u^{\epsilon_{j}} \mathrm{~d} x\right] \\
=\lim _{\epsilon_{j} \rightarrow 0}\left\|a^{\epsilon_{j}}\right\|_{L^{2}(Q)}^{2}+\lim _{\epsilon_{j} \rightarrow 0} \int_{Q} A_{\epsilon_{j}} \nabla a^{\epsilon_{j}} \nabla a^{\epsilon_{j}} \mathrm{~d} x \\
\quad+2 \lim _{\epsilon_{j} \rightarrow 0} \mathbb{E} \int_{0}^{t}\left(f^{\epsilon_{j}}, u_{t}^{\epsilon_{j}}\right) \mathrm{d} s+\lim _{\epsilon_{j} \rightarrow 0} \int_{0}^{t}\left\|g^{\epsilon_{j}}\right\|_{L^{2}(Q)}^{2} .
\end{aligned}
$$

The vanishing of the expectation of the stochastic integrals is due to the fact that $\left(g^{\epsilon_{j}}, u_{t}^{\epsilon_{j}}\right)$ and $\left(g, u_{t}\right)$ are square integrable in time (see assumption (A5) and estimate (1)). Using convergence (38), (51), (52), (53) and (54), we obtain the limits for the terms in the right-hand side of (58). Hence

$$
\begin{aligned}
& \lim _{\epsilon_{j} \rightarrow 0}\left[\mathbb{E}\left\|u_{t}^{\epsilon_{j}}\right\|_{L^{2}(Q)}^{2}+\mathbb{E} \int_{Q} A_{\epsilon_{j}} \nabla u^{\epsilon_{j}} \nabla u^{\epsilon_{j}} \mathrm{~d} x\right] \\
& \quad=\|b(x)\|_{L^{2}(Q)}^{2}+\int_{Q} A_{0} \nabla a(x) \nabla a(x) \mathrm{d} x+2 \mathbb{E} \int_{0}^{t}\left(f, u_{t}\right) \mathrm{d} s+\int_{0}^{t}\|g\|_{L^{2}(Q)}^{2} .
\end{aligned}
$$


Again applying Itô's formula to $\left\|u_{t}\right\|_{L^{2}(Q)}^{2}$ using the homogenized equation, integrating over $(0, t)$ and taking the expectation, we obtain

$$
\begin{aligned}
& \mathbb{E}\left\|u_{t}\right\|_{L^{2}(Q)}^{2}+\mathbb{E} \int_{Q} A_{0} \nabla u \nabla u \mathrm{~d} x \\
& =\|b(x)\|_{L^{2}(Q)}^{2}+\int_{Q} A_{0} \nabla a(x) \nabla a(x) \mathrm{d} x+2 \mathbb{E} \int_{0}^{t}\left(f, u_{t}\right) \mathrm{d} s+\int_{0}^{t}\|g\|_{L^{2}(Q)}^{2} .
\end{aligned}
$$

Now using (59), (60), (50) and (19), we have

$$
\begin{aligned}
\lim _{\epsilon_{j} \rightarrow 0} \mathbb{E} \int_{Q} A_{\epsilon_{j}} \nabla u^{\epsilon_{j}} \nabla u^{\epsilon_{j}} \mathrm{~d} x & =\mathbb{E} \int_{Q \times Y} A(y)\left(\nabla_{x} u(t, x)-\nabla_{y} \chi(y) \nabla_{x} u(t, x)\right) \nabla_{x} u(t, x) \mathrm{d} y \mathrm{~d} x \\
& =\mathbb{E} \int_{Q \times Y}\left(A(y) \nabla_{x} u(t, x)+\nabla_{y} u_{1}(t, x, y)\right) \nabla_{x} u(t, x) \mathrm{d} y \mathrm{~d} x
\end{aligned}
$$

But from (47), we have

$$
\mathbb{E} \int_{Q \times Y}\left(A(y) \nabla u(t, x)+\nabla_{y} u_{1}(t, x, y)\right) \nabla_{y} u_{1}(t, x, y) \mathrm{d} y \mathrm{~d} x=0 .
$$

Therefore (61) and (62) give

$$
\begin{aligned}
& \lim _{\epsilon_{j} \rightarrow 0} \mathbb{E} \int_{Q} A_{\epsilon_{j}} \nabla u^{\epsilon_{j}} \nabla u^{\epsilon_{j}} \mathrm{~d} x \\
& \quad=\mathbb{E} \int_{Q \times Y} A(y)\left[\nabla_{x} u(t, x)+\nabla_{y} u_{1}(t, x, y)\right]\left[\nabla_{x} u(t, x)+\nabla_{y} u_{1}(t, x, y)\right] \mathrm{d} y \mathrm{~d} x .
\end{aligned}
$$

Next, using the two-scale convergence of $\nabla u^{\epsilon_{j}}$, with the test function $A(y)\left(\nabla u(t, x)+\nabla_{y} u_{1}(t, x, y)\right)$, we obtain

$$
\begin{aligned}
& \lim _{\epsilon_{j} \rightarrow 0} \int_{0}^{T} \int_{Q} \nabla u^{\epsilon_{j}}(t, x) A\left(\frac{x}{\epsilon_{j}}\right)\left(\nabla u+\nabla_{y} u_{1}\left(t, x, \frac{x}{\epsilon_{j}}\right)\right) \mathrm{d} x \mathrm{~d} t \\
& \quad=\int_{0}^{T} \int_{Q \times Y}\left(\nabla u(t, x)+\nabla_{y} u_{1}(t, x, y)\right) A(y)\left(\nabla u(t, x)+\nabla_{y} u_{1}(t, x, y)\right) \mathrm{d} x \mathrm{~d} y \mathrm{~d} t .
\end{aligned}
$$

Now, let us write

$$
\begin{aligned}
\psi(t, x, y)= & A(y)\left(\nabla u(t, x)+\nabla_{y} u_{1}(t, x, y)\right) \cdot\left(\nabla u(t, x)+\nabla_{y} u_{1}(t, x, y)\right) \\
= & A(y) \nabla u(t, x) \nabla u(t, x)+2 A(y) \nabla u(t, x) \nabla_{y} u_{1}(t, x, y) \\
& +A(y) \nabla_{y} u_{1}(t, x, y) \nabla_{y} u_{1}(t, x, y) .
\end{aligned}
$$


For $u_{1}$ given by (19), we have

$$
\begin{aligned}
\psi(t, x, y)= & A(y) \nabla u(t, x) \nabla u(t, x)-2 A(y) \nabla u(t, x) \nabla_{y}\left[\chi(y) \cdot \nabla_{x} u(t, x)\right] \\
& +A(y) \nabla_{y}\left[\chi(y) \cdot \nabla_{x} u(t, x)\right] \nabla_{y}\left[\chi(y) \cdot \nabla_{x} u(t, x)\right] .
\end{aligned}
$$

Now using (ii) of Lemma 9, for $p=2$, we obtain

$$
\begin{gathered}
\lim _{\epsilon_{j} \rightarrow 0} \int_{0}^{T} \int_{Q} A\left(\frac{x}{\epsilon_{j}}\right)\left(\nabla u(t, x)+\nabla_{y} u_{1}\left(t, x, \frac{x}{\epsilon_{j}}\right)\right) \cdot\left(\nabla u(t, x)+\nabla_{y} u_{1}\left(t, x, \frac{y}{\epsilon_{j}}\right)\right) \mathrm{d} x \mathrm{~d} t \\
=\int_{0}^{T} \int_{Q \times Y} A(y)\left(\nabla u(t, x)+\nabla_{y} u_{1}(t, x, y)\right) \cdot\left(\nabla u(t, x)+\nabla_{y} u_{1}(t, x, y)\right) \mathrm{d} x \mathrm{~d} y \mathrm{~d} t .
\end{gathered}
$$

Combining (63), (64) and (65) into (57), we deduce that

$$
\lim _{\epsilon_{j} \rightarrow 0} \mathbb{E} \int_{0}^{T}\left\|\nabla u^{\epsilon_{j}}-\nabla u-\nabla_{y} u_{1}\left(\cdot, \cdot, \frac{\cdot}{\epsilon_{j}}\right)\right\|_{L^{2}(Q)}^{2} \mathrm{~d} t=0, \quad \tilde{\mathbb{P}} \text {-a.s. }
$$

Thus the proof is complete.

The asymptotic expansion method seems to be easier than the two scale convergence method. However this is not true of what obtainable in practice, due to the establishing of the expansion (24). Though it allows us to guess the homogenized equation at early stage of the analysis. But more steps and regularity assumptions in the domain as well as in the data are needed to obtain the convergence of the solutions of the original problem to that one of the homogenized problem. Unlike the asymptotic expansion method, the two scale convergence method obtains the homogenization result in only one step. Applying the two scale convergence to (11), we see that the solution of the homogenized problem is in fact the first term of (11), which strongly justifies the well posedness of the multiple expansion method.

As a closing remark, we note that our results can readily be extended to the case of infinite dimensional Wiener processes taking values in appropriate Hilbert spaces; for instance cylindrical Wiener processes.

\section{Acknowledgements}

The authors gratefully acknowledge the financial support of the National Research Foundation of South Africa and the University of Pretoria. They express their gratitude to the reviewers for their careful reading of the paper and useful comments and suggestions.

\section{References}

[1] A. Abdulle and M.J. Grote, Finite element heterogeneous multiscale method for the wave equation, Multiscale Modeling and Simulation 9 (2011), 766-792.

[2] A. Abdulle, M.J. Grote and C. Stohrer, FE heterogeneous multiscale method for long time wave propagation, Comptes Rendus Mathématique (Académie des Sciences) 351 (2013), 495-499.

[3] G. Allaire, Homogenization and two-scale convergence, SIAM J. Math. Anal. 23 (1992), 1482-1518. 
[4] G. Allaire, Two-scale convergence: A new method in periodic homogenization, in: Nonlinear Partial Differential Equations and Their Applications. Collège de France Seminar, Vol. XII, Paris, 1991-1993, Pitman Res. Notes Math. Ser., Vol. 302, Longman Sci. Tech., Harlow, 1994, pp. 1-14.

[5] H. Attouch, Variational Convergence for Functions and Operators, Applicable Mathematics Series, Pitman Advanced Publishing Program, Boston, MA, 1984.

[6] N. Bakhvalov and G. Panasenko, Homogenisation: Averaging Processes in Periodic Media. Mathematical Problems in the Mechanics of Composite Materials, Mathematics and Its Applications (Soviet Series), Vol. 36, Kluwer Academic Publishers, Dordrecht, 1989. (Translated from Russian by D. Leites.)

[7] A. Bensoussan, Homogenization of a class of stochastic partial differential equations, in: Composite Media and Homogenization Theory, Trieste, 1990, Progr. Nonlinear Differential Equations Appl., Vol. 5, Birkhäuser, Boston, MA, 1991, pp. 47-65.

[8] A. Bensoussan, Some existence results for stochastic partial differential equations, in: Stochastic Partial Differential Equations and Applications, Trento, 1990, Pitman Res. Notes Math. Ser., Vol. 268, Longman Sci. Tech., Harlow, 1992, pp. 37-53.

[9] A. Bensoussan, J.-L. Lions and G. Papanicolaou, Asymptotic Analysis for Periodic Structures, AMS Chelsea Publishing, Providence, RI, 2011. (Corrected reprint of the 1978 original.)

[10] P. Billingsley, Convergence of Probability Measures, 2nd edn, Wiley Series in Probability and Statistics, Wiley, 2008.

[11] N. Bleistein, J.K. Cohen and J.W. Stockwell Jr., Mathematics of Multidimensional Seismic Imaging, Migration, and Inversion. Geophysics and Planetary Sciences, Interdisciplinary Applied Mathematics, Vol. 13, Springer, New York, 2001.

[12] A. Bourgeat, A. Mikelić and S. Wright, Stochastic two-scale convergence in the mean and applications, J. Reine Angew. Math. 456 (1994), 19-51.

[13] S. Brahim-Otsmane, G.A. Francfort and F. Murat, Correctors for the homogenization of the wave and heat equations, Journal de Mathématiques Pures et Appliquées 71(3) (1992), 197-231.

[14] D. Cioranescu, A. Damlamian and G. Griso, Periodic unfolding and homogenization, C. R. Acad. Sci. Paris, Ser. 335(1) (2002), 99-104.

[15] D. Cioranescu, A. Damlamian and G. Griso, The periodic unfolding method in homogenization. Multiple scales problems in biomathematics, mechanics, physics and numerics, in: GAKUTO Internat. Ser. Math. Sci. Appl., Vol. 31, Gakkōtosho, Tokyo, 2009, pp. 1-35.

[16] D. Cioranescu and P. Donato, Exact internal controllability in perforated domains, J. Math. Pures Appl. (9) 68(2) (1989), 185-213.

[17] D. Cioranescu and P. Donato, An Introduction to Homogenization, Oxford Lecture Series in Mathematics and Its Applications, Vol. 17, The Clarendon Press, Oxford Univ. Press, New York, 1999.

[18] G. Deugoue and M. Sango, Weak solutions to stochastic 3D Navier-Stokes- $\alpha$ model of turbulence: $\alpha$-asymptotic behavior, J. Math. Anal. Appl. 384(1) (2011), 49-62.

[19] N. Ichihara, Homogenization problem for stochastic partial differential equations of Zakai type, Stoch. Stoch. Rep. 76(3) (2004), 243-266.

[20] L. Jiang, Y. Efendiev and V. Ginting, Analysis of global multiscale finite element methods for wave equations with continuum spatial scales, Appl. Numer. Math. 60(8) (2010), 862-876.

[21] E.Y. Khruslov, Homogenized models of composite media, in: Composite Media and Homogenization Theory, Trieste, 1990, Progr. Nonlinear Differential Equations Appl., Vol. 5, Birkhäuser, Boston, MA, 1991, pp. 159-182.

[22] M.L. Kleptsyna and A.L. Piatnitski, Homogenization of a random non-stationary convection-diffusion problem, Russian Math. Surveys 57 (2002), 729-751.

[23] O. Korostyshevskaya and S.E. Minkoff, A matrix analysis of operator-based upscaling for the wave equation, SIAM J. Numer. Anal. 44(2) (2006), 586-612.

[24] S.M. Kozlov, The averaging of random operators, Mat. Sb. (N. S.) 109(151)(2) (1979), 188-202.

[25] D. Lukkassen, G. Nguetseng and P. Wall, Two-scale convergence, Int. J. Pure Appl. Math. 2(1) (2002), 35-86.

[26] G.D. Maso and L. Modica, Nonlinear stochastic homogenization and ergodic theory, J. Rei. Ang. Math. B. 368 (1986), $27-42$.

[27] F. Murat and L. Tartar, $H$-convergence, in: Topics in the Mathematical Modelling of Composite Materials, A. Cherkaev and R. Kohn, eds, Birkhäuser, Boston, 1977, pp. 21-43.

[28] G. Nguetseng, A general convergence result for a functional related to the theory of homogenization, SIAM J. Math. Anal. 20(3) (1989), 608-623.

[29] G. Nguetseng, Homogenization structures and applications I, Z. Anal. Anwend. 22 (2003), 73-107.

[30] G. Nguetseng, M. Sango and J.L. Woukeng, Reiterated ergodic algebras and applications, Comm. Math. Phys. 300(3) (2010), 835-876.

[31] O.A. Oleinik, A.S. Shamaev and G.A. Yosifian, Mathematical Problems in Elasticity and Homogenization, Studies in Mathematics and Its Applications, Vol. 26, North-Holland, Amsterdam, 1992. 
[32] M. Ondreját, Uniqueness for stochastic evolution equations in Banach spaces, Dissertationes Mathematicae 426 (2004), $1-63$.

[33] H. Owhadi and L. Zhang, Numerical homogenization of the acoustic wave equations with a continuum of scales, Comput. Methods Appl. Mech. Engrg. 198(3,4) (2008), 397-406.

[34] G.C. Papanicolaou and S.R.S. Varadhan, Boundary value problems with rapidly oscillating random coefficients, in: Random Fields, Vols I, II, Esztergom, 1979, Colloq. Math. Soc. János Bolyai, Vol. 27, North-Holland, Amsterdam, 1981, pp. 835-873.

[35] E. Pardoux, Équations aux dérivées partielles stochastiques non linéaires monotones, Thèse, Université Paris XI, 1975.

[36] E. Pardoux and A.L. Piatnitski, Homogenization of a nonlinear random parabolic partial differential equation, Stochastic Process Appl. 104 (2003), 1-27.

[37] P. Razafimandimby, M. Sango and J.L. Woukeng, Homogenization of a stochastic nonlinear reaction-diffusion equation with a large reaction term: The almost periodic framework, J. Math. Anal. Appl. 394(1) (2012), 186-212.

[38] P.A. Razafimandimby and M. Sango, Asymptotic behavior of solutions of stochastic evolution equations for second grade fluids, C. R. Math. Acad. Sci. Paris 348(13,14) (2010), 787-790.

[39] P.A. Razafimandimby and M. Sango, Convergence of a sequence of solutions of the stochastic two-dimensional equations of second grade fluids, Asymptot. Anal. 79(3,4) (2012), 251-272.

[40] P.A. Razafimandimby and J.L. Woukeng, Homogenization of nonlinear stochastic partial differential equations in a general ergodic environment, Stoch. Anal. Appl. 31(5) (2013), 755-784.

[41] E. Sanchez-Palencia, Non-Homogeneous Media and Vibration Theory, Lecture Notes in Physics, Springer, 1980.

[42] E. Sanchez-Palencia and A. Zaoui, Homogenization Techniques for Composite Media: Lectures Delivered at the Cism International Center for Mechanical Sciences, Udine, Italy, July 1-5, 1985, Lecture Notes in Physics, 1985.

[43] M. Sango, Asymptotic behavior of a stochastic evolution problem in a varying domain, Stochastic Anal. Appl. 20(6) (2002), 1331-1358.

[44] M. Sango, Splitting-up scheme for nonlinear stochastic hyperbolic equations, Forum Math. 25(5) (2013), 931-965.

[45] M. Sango, Homogenization of stochastic semilinear parabolic equations with non-Lipschitz forcings in domains with fine grained boundaries, Commun. Math. Sci. 12(2) (2014), 345-382.

[46] M. Sango, N. Svanstedt and J.L. Woukeng, Generalized Besicovitch spaces and applications to deterministic homogenization, Nonlinear Anal. 74(2) (2011), 351-379.

[47] M. Sango and J.L. Woukeng, Stochastic two-scale convergence of an integral functional, Asymptot. Anal. 73(1,2) $(2011)$, 97-123.

[48] M. Sango and J.L. Woukeng, Stochastic $\Sigma$-convergence and applications, Dyn. Partial Differ. Equ. 8(4) (2011), 261-310.

[49] J. Simon, Compact sets in the space $L_{p}(0, T ; B)$, Ann. Mat. Pura Appl., IV. Ser. 146 (1987), 65-96.

[50] N. Svanstedt, Multiscale stochastic homogenization of monotone operators, Netw. Heterog. Media 2(1) (2007), 181-192.

[51] L. Tartar, Quelques remarques sur l'homogénésation, in: Functional Analysis and Numerical Analysis, Proc. JapanFrance Seminar 1976, H. Fujita, ed., Japanese Society for the Promotion of Science, 1977, pp. 468-486.

[52] L. Tartar, Compensated compactness and applications to partial differential equations, in: Nonlinear Analysis and Mechanics. Heriot Watt Symposium, Vol. IV, R.J. Knops, ed., Research Notes in Mathematics, Vol. 39, Pitman, 1979, pp. 136-212.

[53] L. Tartar, The General Theory of Homogenization. A Personalized Introduction, Lecture Notes of the Unione Matematica Italiana, Vol. 7, Springer, Berlin; UMI, Bologna, 2009.

[54] J.B. Walsh, An introduction to stochastic partial differential equations, in: Lecture Notes in Math., Vol. 1180, Springer, Berlin, 1986, pp. 265-439. 